AN ASSESSMENT OF IOW FLOWS IN STREAMS

IN NORTHEASTERN WYOMING

By G.W. Armentrout, Jr. and James F. Wilson, Jr.

U.S. GEOLOGICAL SURVEY

Water-Resources Investigations Report 85-4246

Cheyenne, Wyaming

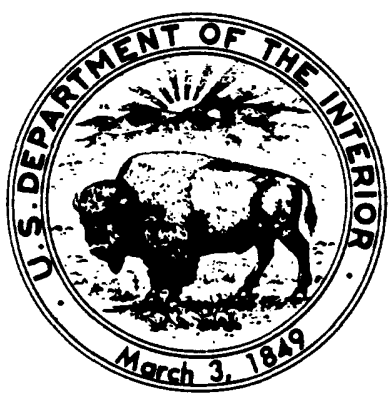




\section{DEPARTMENT OF THE INIERIOR \\ DONALD PAUL HODEL, Secretary \\ U.S. GEOLOGICAL SURVEY}

Dallas L. Peck, Director

For additional informaton write to:

District Chief

U.S. Geological Survey

2120 Capitol Avenue

P.O. Box 1125

Cheyenne, Wyaming 82003
Copies of this report can be purchased from:

U.S. Geological Survey Books and Open-File Reports Federal Center, Box 25425

Denver, Colorado 80225 


$\begin{array}{lr}\text { Abstract- } & 1 \\ \text { Introduction- } & 2 \\ \text { Purpose and scope- } & 2 \\ \text { Use of low-flow information- } & 4 \\ \text { Previous investigations- } & 4 \\ \text { Description of the area- } & 5 \\ \text { Low-flow characteristics at stations- } & 6 \\ \text { Data available- } & 6 \\ \text { Minimum observed flows- } & 13 \\ \text { Flow-duration curves } & 13 \\ \text { Frequency analysis- } & 15 \\ \text { Seven-day low flows- } & 15 \\ \text { No-flow periods- } & 19 \\ \text { Low-flow characteristics at ungaged sites- } & 19 \\ \text { Mountain streams- } & 22 \\ \text { Selected main-stem streams- } & 23 \\ \text { Discussion- } & 25 \\ \text { Accuracy of results- } & 25 \\ \text { Future investigations- } & 26 \\ \text { References cited- } & 28\end{array}$

PLATES

Plate 1. Map showing location of stations and miscellaneousobservation sites

In pocket

FIGURES

Figure 1. Map showing location of the study area and principal drainage basins

2. Typical hydrographs of daily discharge for an ephemeral stream and a perennial stream in northeastern Wyaming (1979 water year)

3. Flow-duration curves for a mountain stream and a plains stream in northeastern Wyoming-

4. Low-flow frequency curves for a mountain stream and a plains stream in northeastern wyoming-

5. Frequency curves for annual maximum number of consecutive days of no flow at five stations on plains streams in northeastern Wyaming and adjacent parts of Montana

6-8. Graphs showing relation of 7-day 10-year low flow to:

6. Drainage area, for streams in the Bighorn Mountains in Wyoming

7. Distance along channels of the Powder River upstream from Moorhead, Mont., and Clear Creek upstream from its mouth at the Powder River

8. Distance along channel, North Platte River between Grey Reef Dam and Guernsey Dam, Wyoming 
Table 1. Streamflow stations and sites included in

Page assessment of low flows

2. Frequency distribution of minimum average 7-day

flows at selected streanflow stations with sufficient records to include a drought, in northeastern Wyaming and adjacent parts of Montana and South Dakota-

3. Seven-day 10-year low flows and frequency of no

flow for selected periods of consecutive days, at selected stations on plains and foothills streams in northeastern Wyoming and adjacent parts of Montana and South Dakota

\section{CONVERSION FACTORS AND VERTICAL DATUM}

The following factors may be used to convert the inch-pound units used in this report to metric units:

\section{Multiply}

cubic foot per second

foot

inch

mile

square mile
By

0.02832

0.3048

2.540

1.609

2.590
To obtain

cubic meter per second meter centimeter kilameter square kilometer

Sea level: In this report "sea level" refers to the National Geodetic Vertical Datum of 1929 (NGVD of 1929)-a geodetic datum derived from a general adjustment of the first-order level nets of both the United States and Canada, formerly called "Mean Sea Level of 1929." 


\title{
AN ASSESSMENT OF LOW FLOWS IN STREAMS IN NORIHEASTERN WYOMING
}

By G. W. Armentrout, Jr. and James F. Wilson, Jr.

\begin{abstract}
This report is a brief summary and assessment of low flows in the following basins in northeastern Wyoming: Little Bighorn, Tongue, Powder, Little Missouri, Belle Fourche, Cheyenne, and Niobrara Rivers, and about 200 river miles of the North Platte River and its tributaries. Only existing data from streamflow stations and miscellaneous-observation sites during the period, 1930-80, were used. Data for a few stations in Montana and South Dakota were used in the analysis. Data were available for 56 perennial streams, 38 intermittent streams, and 34 ephemeral streams.

The distribution of minimum observed flows of record at all stations and sites and the 7-day 10-year low flows at mountain stations and main-stem plains stations are shown on a map. Seven-day low flows were determined by fitting the log Pearson Type III distribution to the data; results are tabulated only for stations with at least 10 years of record that included at least one major drought. Stations installed since about 1960 are considered not to have included a major drought. Most streams that originate in the foothills and plains have no flow during part of every year, and are typical of much of the study area. For stations on these streams, the frequency of the annual maximum number of consecutive days of no flow was determined, as an indicator of the likelihood of extended periods of no flow or drought.

For estimates at ungaged sites on streams in the Bighorn Mountains only, a simple regression of 7-day 10-year low flow on drainage area has a standard error of 64 percent, based on 19 stations with drainage areas of 2 to 200 square miles. The 7-day 10year low flow in main-stem streams can be interpolated from graphs of 7-day 10-year low flow versus distance along the main channel.

Additional studies of low flow are needed. The data base, particularly synoptic baseflow information, needs considerable expansion. Also, the use of storage-analysis procedures should be considered as a means of assessing the availability of water in streams that otherwise are fully appropriated or that are ephemeral.
\end{abstract}




\section{INIRODUCTION}

As the development of coal and other energy minerals has increased in northeastern Wyoming there has been a corresponding increase in the demand for water. Ground water is the principal source for domestic, stock, municipal, and industrial use; surface water is the principal source for irrigation, the largest use of water. Streams in the mountains that border the study area (fig. 1) and a few large streams that flow across the area either are perennial or flow for extended periods during most years. Water in those streams generally is fully appropriated. Most plains streams in the central part of the area flow only in response to precipitation; these ephemeral streams are dry most of the time. Because the availability of surface water is so limited, planners and others concerned with water use need additional information for assessing long-term supplies.

One means of assessing the potential supplies of limited surface-water resources is a low-flow analysis--a statistical evaluation of minimum streamflows observed at gaging stations and miscellaneous-observation sites. Such an analysis provides information about existing flows that are an integral part of the hydrologic system, support the present ecologic system, and support and are affected by the humans who use the water. The study of minimum flows in streams of northeastern Wyoming was done during 1982. This report describes the results of the study, which was part of the U.S. Geological Survey's Coal-Hydrology Program.

\section{Purpose and Scope}

The purpose of this report is to summarize and assess selected low-flow characteristics of streams in northeastern Wyaming (fig. 1 and plate 1 ), as a basis for more detailed low-flow studies in the future. Low-flow characteristics are indicators of the amount of water available under present conditions--that is, without considering the use of artificial storage. The study was not intended to be an in-depth treatment of low flows in the area. Possible future studies are discussed at the end of this report.

Only existing data from Geological Survey streamflow stations and miscellaneous streamflow-observation sites through water year 1980 were used in the analysis. A few stations in Montana and South Dakota were used, to extend the analysis across the State lines. The period of record was used as a criterion to select the stations used in the analysis. In general, standard low-flow frequency-analysis techniques were used to analyze records for stations on mountain streams and main stems of plains streams fed by mountain tributaries. Standard techniques, however, cannot be applied to records of flows in ephemeral streams that are typical of much of the study area. For those streams, which are dry most of the time, the number of consecutive days of no flow was analyzed. 


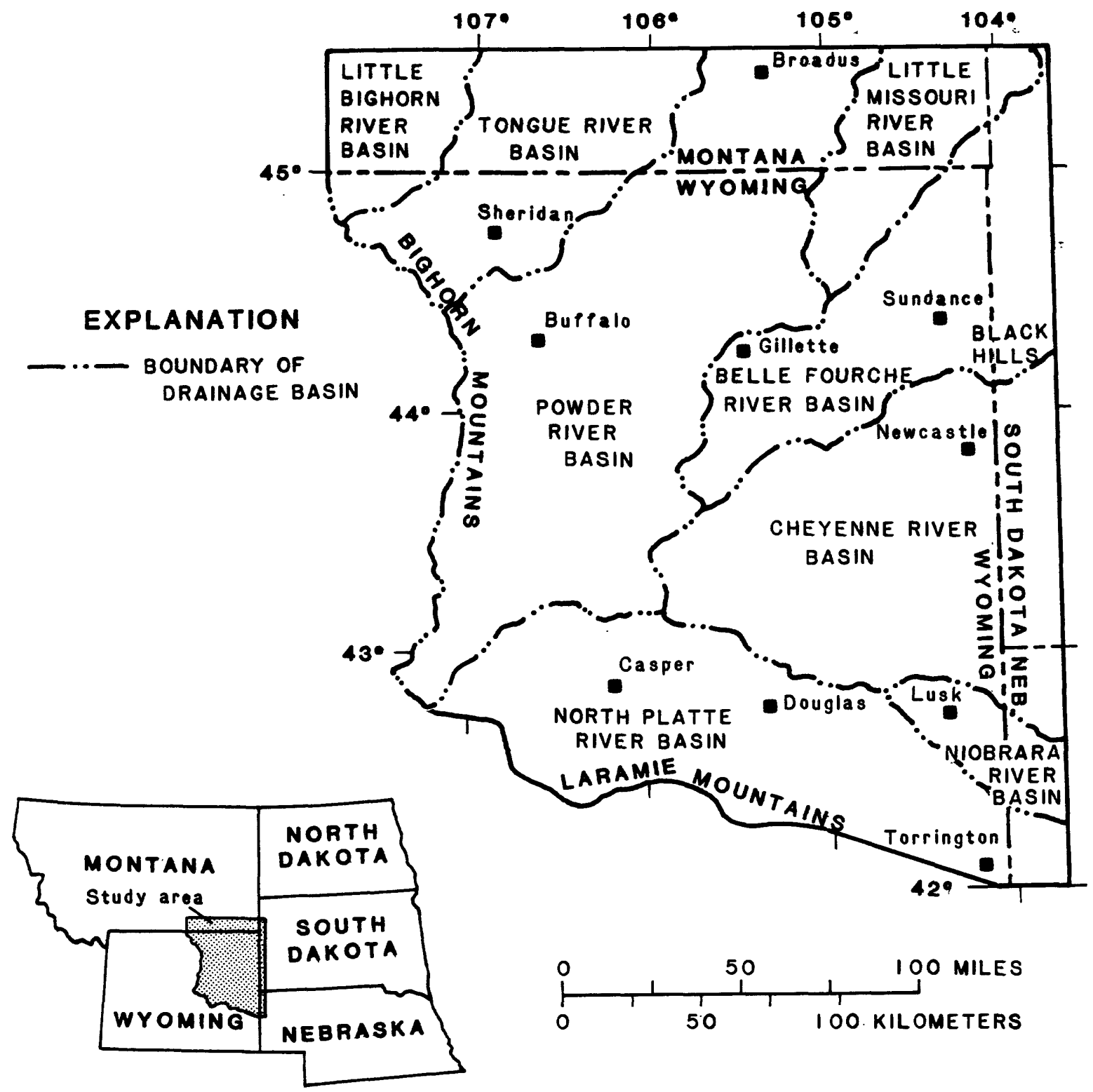

Figure 1.--Location of the study area and principal river basins. (Eight streamflow stations in adjacent States were used in the study.) 


\section{Use of Low-Flow Information}

Low-flow information is useful for: (1) Planning and design of water supplies, (2) analysis of environmental and economic consequences of human activities, (3) modeling water-quality processes in streams, and (4) determination of optimum and maintenance flows for instream uses (Riggs, 1980, p. 728). According to Chiang and Johnson (1976, p. 227), "If the low flow capacity of a stream is not adequate to satisfy all the demands for its water, including the environmental and recreational needs for in-stream uses, the matter of priorities becomes a paramount and difficult issue." Riggs (1980, p. 717) describes the degradation in water quality as streamflow decreases in a given reach, and states: "The natural or existing low-flow characteristics of a stream, or both, should be considered in establishing a specified flow to be maintained for one or more purposes."

Low-flow information can be used to assess the possibility of drought, or insufficient flow for the intended uses of the water. Sen (1980, p. 99) stressed the importance of considering the possibility of drought conditions during the projected life of a water-resources system. For northeastern Wyoming, the assessment of existing low flows and the possibility of drought can be useful in determining: (1) The need for supplies for supplemental watering of stock, (2) the frequency of flushing of ephemeral streams for dilution and disposal of wastes, and (3) the consequences of not building structures, such as stream-bypass canals and detention ponds, for new or expanded mines, mills, and energy plants. In this report the potential for drought is indicated by the frequency of periods of no flow in ephemeral plains streams.

\section{Previous Investigations}

Basic references on the subject of low-flow investigations include: An evaluation of various definitions of baseflow recession (Singh, 1969), a discussion of the probability distribution of low flow (Matalas, 1963), a description of standard methods of low-flow analysis used by the Geological Survey (Riggs, 1972), and a summary of approaches most used by government agencies, consulting firms, universities, and utility companies (Riggs, 1980).

Although there is little difficulty in computing low-flow characteristics for individual streamflow stations, estimating low flows at ungaged sites is quite another matter. The technique most often used (called regionalization) is some variation of mathematical regression. The results commonly are less than satisfactory, mainly because of the difficulty in determining the independent variables that affect low flows and in defining those variables quantitatively. Consequently, regionalization of low flows in various parts of the United States and in other countries is characterized by a wide variety of approaches. Selected references on the subject include: Furness (1960), Furness and Busby (1967), Singh and Stall (1974), Skelton (1974), Chang and Boyer (1977), Huntzinger (1978), Institute of Hydrology (1979), Lara (1979), and Bingham (1982). In general, the farther east the study area, the more accurate were the results. Some interesting studies that did not include regionalization were those by Speer and others (1964, 1965) and by Chiang and Johnson (1976). 
The only previous comprehensive analysis of low-flow information for Wyaning streams was done by Wahl (1970). Wahl's study was a part of a nationwide assessment of streamflow data on a state-by-state basis. The study included regressions of low-flow characteristics on basin characteristics. Even for mountain streams, the accuracy of the regression equations for low flows failed by a substantial margin to meet arbitrary accuracy goals based on streamflow variability. This finding was common in similar reports for other States. Wahl concluded that additional data were needed and that alternative methods for regionalizing low-flow data also were needed.

Since 1970 the streamflow data base for northeastern Wyoming has been increased substantially, particularly for small, ephemeral streams. In response to energy-resource development many new stations were established on small streams. Also, during 1977-78 a set of base-flow measurements was made at more than 100 miscellaneous sites in northeastern Wyoming (Druse and others, 1981).

Studies of the frequency, duration, and other characteristics of droughts may be useful to those considering the results of low-flow analyses. Selected references on that subject are: Huff and Changenon (1964), Whipple (1966), and Sen (1980). A detailed report of the drought of 1976-77 in the central and western United States was prepared by Matthai (1979).

\section{DESCRIPTION OF THE AREA}

The study area, which includes all of northeastern wyoming (fig. I and plate 1), extends from the Laramie Mountains northward into Montana, and from the Bighorn Mountains eastward into South Dakota. Low-flow data for selected stations near the Wyoming border in Montana and South Dakota were used in the study. The area in Wyoming includes all of the following basins: Little Bighorn, Tongue, Powder, Little Missouri, Belle Fourche, Cheyenne, and Niobrara Rivers, and about 200 river miles of the North Platte River and its tributaries (about 7 percent of its total drainage area) between Alcova and Guernsey Reservoirs.

For purposes of hydrologic analysis, the study area was subdivided into two geographic types--mountains and plains. Mountain areas were defined as those higher than about 7,500 feet above sea level in the Laramie Mountains and southern Bighorn Mountains; higher than 5,000 feet in the northern Bighorn Mountains; and higher than 5,000 to 5,500 feet in the Black Hills. The remainder of the area, including the lower foothills adjacent to the mountains, was classified as plains.

Average annual precipitation is 15 to 25 inches in the Bighorn Mountains, 15 to 24 inches in the Black Hills, and 12 to 14 inches in the Laramie Mountains. Most of the study area consists of plains, where the average annual precipitation is 12 to 14 inches. In some parts of the area as little as 7 inches of annual precipitation has been recorded. 
There are many reports that describe the hydrology of the area. Hodson and others (1973) describe the water resources (especially ground water) of northeastern Wyaming. Lowry, Wilson, and others (1986) describe the hydrology of the Powder River basin in relation to development of the area's large deposits of coal; the report includes a bibliography of more than 350 references. Methods for estimating average annual discharge and flood-flow characteristics at ungaged sites are given in a report by Lowham (1976); a report on revised methods is planned for publication in 1987. Evapotranspiration, an important factor affecting low flows, was estimated at several sites in the Powder River basin by Lenfest (1986).

\section{LOW-FLOW CHARACTERISTICS AT STATIONS}

\section{Data Available}

Streamflow records at Geological Survey gaging stations and observations at miscellaneous sites were used in the analysis. There are two types of gaging stations: continuous-record stations, at which the discharge is monitored with a recorder, and partial-record stations, at which only the annual maximum discharge generally is determined. A few high-flow partial-record stations (no longer in operation) were equipped with hydrograph recorders that were operated during May-September each year. Annual minimum flows were determined at recording stations, and when observed, no flow was noted at partial-record and nonrecording stations and miscellaneous sites.

Prior to about 1972 most continuous-record stations in the area were located on streams flowing from the mountains and on the few large rivers that are fed by mountain streams and flow across the plains. Between 1972 and 1980 many new stations were installed in the central part of the area, particularly on small plains streams, as part of the Geological Survey and U.S. Bureau of Land Management coal-hydrology programs. Although the new stations have added substantially to the data base available for low-flow analysis, their short length of record limits the usefulness of the data for detailed analysis.

The stations and sites used in the analysis are listed in downstream order in table 1; the locations are shown in plate I (in pocket). Although the permanent 8-digit station numbers used by the Geological Survey are listed in table 1, a sequential identification number (1-128) is used throughout this report to identify stations and sites.

Data were available for three types of streams: (1) Perennial streams, which flow continuously; (2) intermittent streams, which flow only at certain times of the year when the stream receives water from springs or some surface source such as melting snow; and (3) ephemeral streams, which flow only in direct response to precipitation, and whose channel is at all times above the water table. The hydrographs of daily discharge for an ephemeral stream and a perennial stream (fig. 2) illustrate why the analysis of low flows differs for these two types of streams. The distribution of stations and sites by stream type is sumarized as follows: 


\begin{tabular}{lcccc}
\hline & \multicolumn{3}{c}{ Number of stations/sites } \\
\cline { 2 - 5 } Station/site type & \multicolumn{4}{c}{ Stream type } \\
\cline { 2 - 5 } & Perennial & Intermittent & Ephemeral & Total \\
\hline Continuous record & 54 & 30 & 12 & 96 \\
Partial record & 2 & - & 17 & 26 \\
Miscellaneous site & - & 37 & 6 & 6 \\
\cline { 2 - 5 } & 56 & 56 & 35 & 128 \\
\hline
\end{tabular}

Data for low-flow studies should include a period of time that includes at least one drought or very dry period. Low-flow characteristics from frequency curves are most reliable when the record is long and homogeneous; a record of 15-20 years may not contain a representative sample of low flows (Riggs, 1980, p. 724). Also, long-term records for streams in the Rocky Mountain area indicate that streamflow prior to 1930 was significantly larger than that since 1930 (the reason for the change is not known). Therefore, except for observations of no flow and (or) minimum flow of record, records for many of the stations and sites listed in table 1 were not analyzed further.

An attempt was made to correlate short-term records for a few stations on the north flank of the Laramie Mountains with long-term records at nearby stations. The correlation failed, probably because the short-term records began during the early 1970's; the subsequent period generally was one of high flows, while the period of record at long-term stations includes at least one major drought. As explained by Riggs (1972, p. 6-9), low flows may be from a streamflow population different from that of the rest of the record at a station.

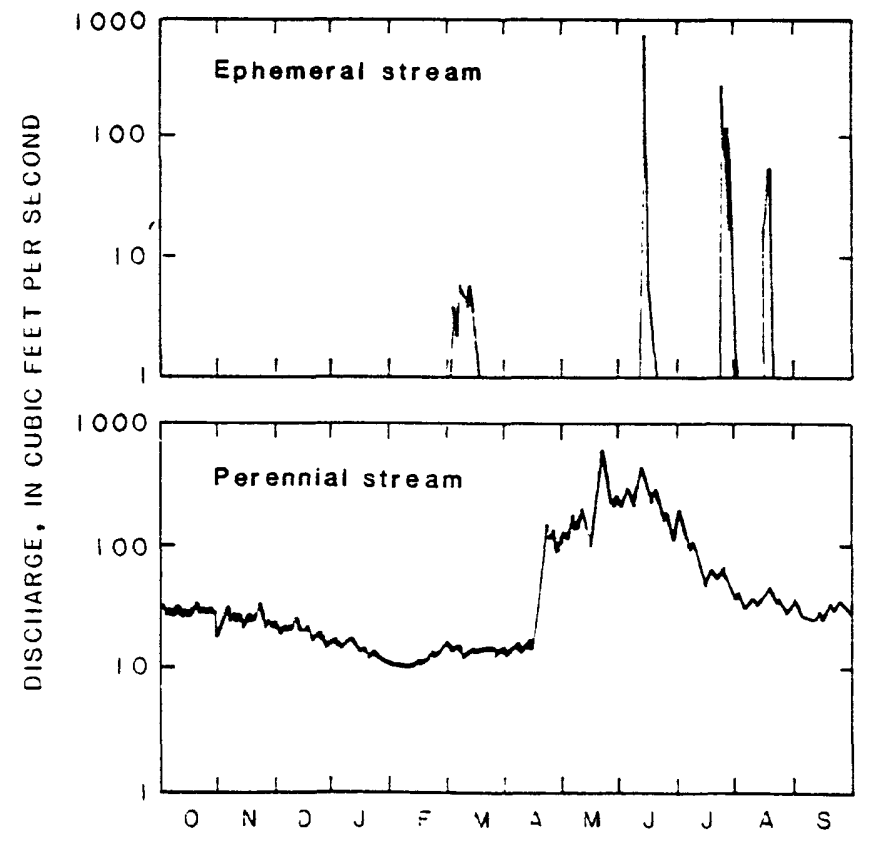

Figure 2.--Typical hydrographs of daily discharge for an ephemeral stream and a perennial stream in northeastern Wyoming (1979 water year).

From Lowry, Wilson, and others (1986, p. 43). 
Table 1.--Streamflow stations and sites included in assessment of lov flows

[ID no: Station or site identification number used in this report;

Record type: Con, continuous; PR, partial record; Misc, miscellaneous;

Stream type: Per, perennial; Int, intermittent; Eph, ephemeral]

\begin{tabular}{|c|c|c|c|c|c|}
\hline $\begin{array}{l}\text { Permanent } \\
\text { ID station } \\
\text { ne. number }\end{array}$ & Station name & $\begin{array}{l}\text { Post }-1929 \\
\text { period } \\
\text { of } \\
\text { record }\end{array}$ & $\begin{array}{c}\text { Record } \\
\text { type }\end{array}$ & $\begin{array}{c}\text { Stream } \\
\text { type }\end{array}$ & $\begin{array}{c}\text { Drainage } \\
\text { area } \\
\text { (square } \\
\text { miles) }\end{array}$ \\
\hline
\end{tabular}

\section{Little Bighorn River Basin}

106289000 Little Bighorn River at State line 1940-80 near Wyola, Mont.

Con Per 193

\section{Tongue River Basin}

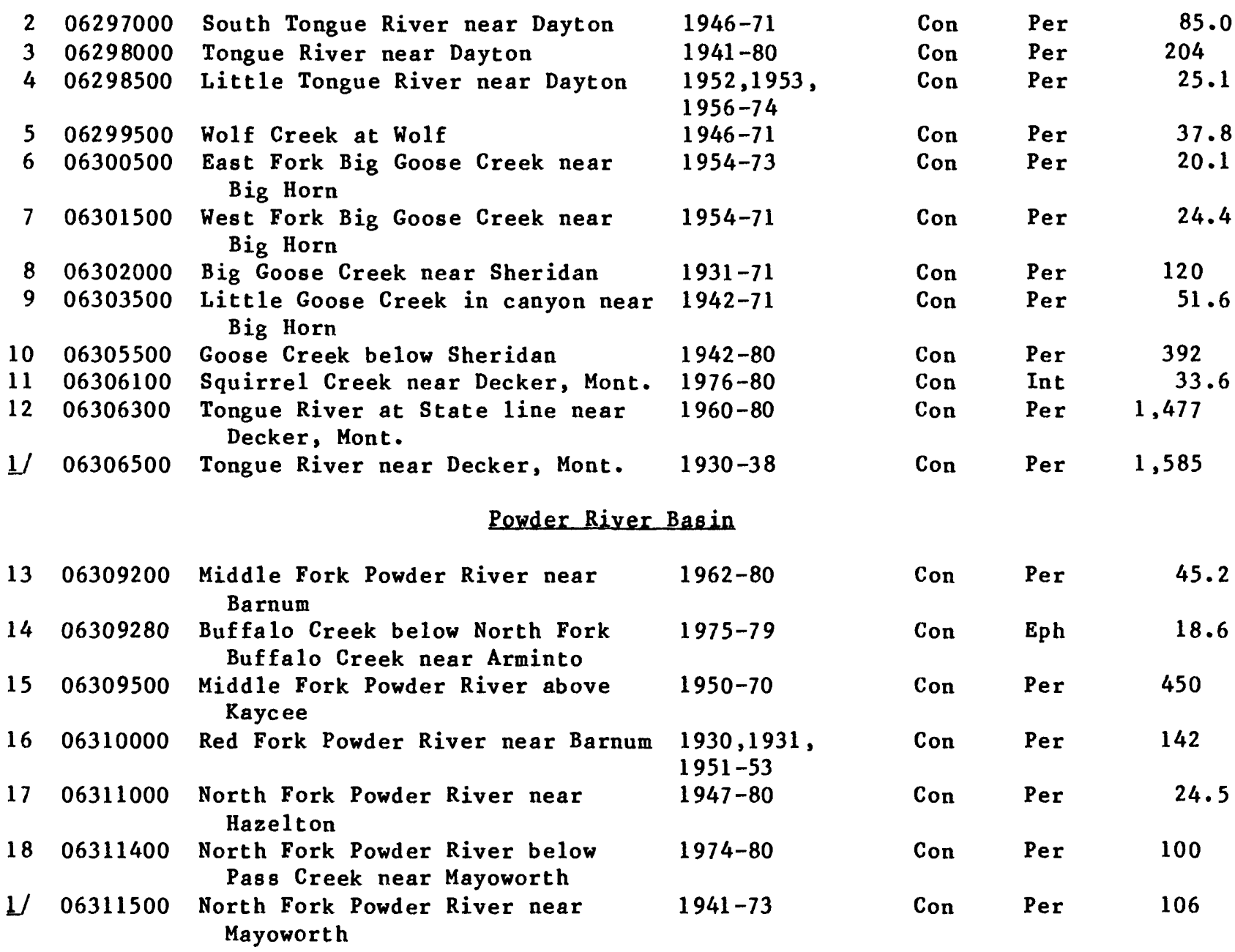


Table 1.--Streamflow stations and sites included in assessment of low flows--Cont inued

\begin{tabular}{|c|c|c|c|c|c|c|}
\hline $\begin{array}{l}\text { ID } \\
\text { ne. }\end{array}$ & $\begin{array}{l}\text { Permanent } \\
\text { station } \\
\text { number }\end{array}$ & Station name & $\begin{array}{l}\text { Post }-1929 \\
\text { period } \\
\text { of } \\
\text { record }\end{array}$ & $\begin{array}{l}\text { Rec ord } \\
\text { type }\end{array}$ & $\begin{array}{c}\text { Stream } \\
\text { type }\end{array}$ & $\begin{array}{c}\text { Drainage } \\
\text { area } \\
\text { (square } \\
\text { miles) }\end{array}$ \\
\hline \multicolumn{7}{|c|}{ Powder River Basin--Continued } \\
\hline 19 & 06312000 & $\begin{array}{l}\text { North Fork Powder River near } \\
\text { Kaycee }\end{array}$ & 1931 & Con & Per & 244 \\
\hline 20 & 06312500 & Powder River near Kaycee & $\begin{array}{l}1939,1941-71 \\
1979,1980\end{array}$ & Con & Per & 980 \\
\hline 21 & 06312700 & $\begin{array}{l}\text { South Fork Powder River near } \\
\text { Powder River }\end{array}$ & $1961-80$ & PR & Int & 262 \\
\hline 22 & 06312795 & $\begin{array}{l}\text { Sanchez Creek above reservoir } \\
\text { near Arminto }\end{array}$ & $1970-80$ & PR & Eph & 5.53 \\
\hline 23 & 06312910 & $\begin{array}{l}\text { Dead Horse Creek tributary near } \\
\text { Midwest }\end{array}$ & $1965-72$ & PR & Eph & 1.53 \\
\hline 24 & 06313000 & $\begin{array}{l}\text { South Fork Powder River near } \\
\text { Kaycee }\end{array}$ & $\begin{array}{l}1939,1951-69 \\
1979,1980\end{array}$ & Con & Int & 1,150 \\
\hline 25 & 06313020 & Bobcat Creek near Edgerton & $1965-80$ & PR & Int & 8.29 \\
\hline 26 & 06313050 & East Teapot Creek near Edgerton & $1965-73$ & PR & Eph & 5.44 \\
\hline 27 & -- & Salt Creek at Midwest & (pre-1930) & Misc & Eph & -- \\
\hline 28 & 06313100 & Coal Draw near Midwest & $1961-80$ & PR & Int & 11.4 \\
\hline 29 & 06313180 & $\begin{array}{l}\text { Dugout Creek tributary near } \\
\text { Midwest }\end{array}$ & $1974-80$ & Con & Eph & .8 \\
\hline 30 & 06313400 & Salt Creek near Sussex & $1977-80$ & Con & Per & 765 \\
\hline 31 & 06313450 & North Spring Draw near Sussex & 1980 & PR & Eph & 5.21 \\
\hline 32 & 06313500 & Powder River at Sussex & $\begin{array}{l}1939,1951-57 \\
1978-80\end{array}$ & Con & Per & 3,090 \\
\hline $\begin{array}{l}33 \\
34\end{array}$ & $\begin{array}{l}06313700 \\
06314000\end{array}$ & $\begin{array}{l}\text { Dead Horse Creek near Buffalo } \\
\text { North Fork Crazy Woman Creek near } \\
\text { Buffalo }\end{array}$ & $\begin{array}{l}1958-71,1971-80 \\
1943-49, \\
1974-80\end{array}$ & $\begin{array}{l}\text { Con } \\
\text { Con }\end{array}$ & $\begin{array}{l}\text { Int } \\
\text { Per }\end{array}$ & $\begin{aligned} 151 \\
44.9\end{aligned}$ \\
\hline 35 & 06314500 & $\begin{array}{l}\text { North Fork Crazy Woman Creek } \\
\text { below Spring Draw near Buffalo }\end{array}$ & $1949-71$ & Con & Per & 51.7 \\
\hline 36 & 06315000 & $\begin{array}{l}\text { North Fork Crazy Woman Creek near } \\
\text { Greub }\end{array}$ & $1951-68$ & Con & Per & 174 \\
\hline 37 & 06315500 & $\begin{array}{l}\text { Middle Fork Crazy Woman Creek } \\
\text { near Greub }\end{array}$ & $1943-71$ & Con & Per & 82.7 \\
\hline $\begin{array}{l}38 \\
39\end{array}$ & $\begin{array}{l}06316000 \\
06316400\end{array}$ & $\begin{array}{l}\text { Crazy Woman Creek near Buffalo } \\
\text { Crazy Woman Creek at upper } \\
\text { station near Arvada }\end{array}$ & $\begin{array}{l}1931-32 \\
1964-70,1977-80\end{array}$ & $\begin{array}{l}\text { Con } \\
\text { Con }\end{array}$ & $\begin{array}{l}\text { Per } \\
\text { Per }\end{array}$ & $\begin{array}{l}464 \\
945\end{array}$ \\
\hline$\frac{1 /}{40}$ & $\begin{array}{l}06316500 \\
06316480\end{array}$ & $\begin{array}{l}\text { Crazy Woman Creek near Arvada } \\
\text { Headgate Draw at upper station } \\
\text { near Buffalo }\end{array}$ & $\begin{array}{l}1940-43,1951-64 \\
1965-73\end{array}$ & $\begin{array}{l}\text { Con } \\
\text { PR }\end{array}$ & $\begin{array}{l}\text { Per } \\
\text { Eph }\end{array}$ & $\begin{array}{l}956 \\
3.32\end{array}$ \\
\hline 41 & 06317000 & Powder River at Arvada & $1931-33,1935-80$ & Con & Per & 6,050 \\
\hline 42 & 06317050 & Rucker Draw near Spotted Horse & $1961-80$ & PR & Eph & 3.98 \\
\hline 43 & 06317500 & $\begin{array}{l}\text { North Fork Clear Creek near } \\
\text { Buffalo }\end{array}$ & $1950-68$ & Con & Per & 29.0 \\
\hline $\begin{array}{l}44 \\
45\end{array}$ & $\begin{array}{l}06318500 \\
06319500\end{array}$ & $\begin{array}{l}\text { Clear Creek near Buffalo } \\
\text { South Fork Rock Creek near } \\
\text { Buffalo }\end{array}$ & $\begin{array}{l}1939-80 \\
1942-48,1951-53\end{array}$ & $\begin{array}{l}\text { Con } \\
\text { Con }\end{array}$ & $\begin{array}{l}\text { Per } \\
\text { Per }\end{array}$ & $\begin{array}{l}120 \\
43.8\end{array}$ \\
\hline
\end{tabular}


Table 1.--Streamflow stations and sites included in assessment of low flows--Continued

\begin{tabular}{|c|c|c|c|c|c|c|}
\hline $\begin{array}{l}\text { ID } \\
\text { ne. }\end{array}$ & $\begin{array}{l}\text { Permanent } \\
\text { station } \\
\text { number }\end{array}$ & Station name & $\begin{array}{l}\text { Post-1929 } \\
\text { period } \\
\text { of } \\
\text { record }\end{array}$ & $\begin{array}{l}\text { Record } \\
\text { type }\end{array}$ & $\begin{array}{c}\text { Stream } \\
\text { type }\end{array}$ & $\begin{array}{c}\text { Drainage } \\
\text { area } \\
\text { (square } \\
\text { miles) }\end{array}$ \\
\hline \multicolumn{7}{|c|}{ Powder River Basin--Continued } \\
\hline 46 & 06320000 & Rock Creek near Buffalo & $1944,1946-71$ & Con & Per & 60.0 \\
\hline $\begin{array}{l}47 \\
48\end{array}$ & $\begin{array}{l}06321500 \\
06323000\end{array}$ & $\begin{array}{l}\text { North Piney Greek near Story } \\
\text { Piney Creek at Kearney }\end{array}$ & $\begin{array}{l}1952-80 \\
1941-80\end{array}$ & $\begin{array}{l}\text { Con } \\
\text { Con }\end{array}$ & $\begin{array}{l}\text { Per } \\
\text { Per }\end{array}$ & $\begin{array}{l}36.8 \\
118\end{array}$ \\
\hline 49 & 06323500 & Piney Creek at Ucross & $1951-80$ & Con & Per & 267 \\
\hline 50 & 06324000 & Clear Creek near Arvada & $1940-80$ & Con & Per & 1,110 \\
\hline 51 & 06324500 & Powder River at Moorhead, Mont. & $1930-72,1975-80$ & Con & Per & 8,088 \\
\hline 52 & 06324890 & $\begin{array}{l}\text { Little Powder River below Corral } \\
\text { Creek near Weston }\end{array}$ & $1978-80$ & Con & Int & 204 \\
\hline $\begin{array}{l}53 \\
54\end{array}$ & $\begin{array}{l}06324925 \\
06324970\end{array}$ & $\begin{array}{l}\text { Little Powder River near Weston } \\
\text { Little Powder River above Dry }\end{array}$ & $\begin{array}{l}1978-80 \\
1973-80\end{array}$ & $\begin{array}{l}\text { Con } \\
\text { Con }\end{array}$ & $\begin{array}{l}\text { Int } \\
\text { Int }\end{array}$ & $\begin{array}{r}540 \\
1,235\end{array}$ \\
\hline & & Creek near Weston & & & & \\
\hline 55 & 06324995 & Badger Creek at Biddle, Mont. & $1972-80$ & PR & Eph & 6.06 \\
\hline 56 & 06325000 & $\begin{array}{l}\text { Little Powder River at Biddle, } \\
\text { Mont. }\end{array}$ & $1939-42$ & Con & Int & 1,540 \\
\hline 57 & 06325500 & $\begin{array}{l}\text { Little Powder River near } \\
\text { Broadus, Mont. }\end{array}$ & $\begin{array}{l}1948-53,1958-61 \\
1963-72\end{array}$ & , Con & Int & 1,974 \\
\hline \multicolumn{7}{|c|}{ Little Missouri River Basin } \\
\hline 58 & 06334000 & $\begin{array}{l}\text { Little Missouri River near } \\
\text { Alzada, Mont. }\end{array}$ & $1930-32,1936-69$ & Con & Int & 904 \\
\hline \multicolumn{7}{|c|}{ Cheyenne River Bas in } \\
\hline 59 & 06363700 & Porcupine Creek near Turnercrest & $1959-76$ & $\mathbf{P R}$ & Eph & 31.5 \\
\hline 60 & 06365300 & Dry Fork Cheyenne River near Bill & $1978-80$ & Con & Int & 128 \\
\hline 61 & 06365900 & Cheyenne River near Dull Center & $1977-80$ & Con & Int & 1,527 \\
\hline 62 & 06375600 & $\begin{array}{l}\text { Little Thunder Creek near } \\
\text { Hampshire }\end{array}$ & $1978-80$ & Con & Eph & 234 \\
\hline 63 & 06376300 & $\begin{array}{l}\text { Black Thunder Creek near } \\
\text { Hampshire }\end{array}$ & $1973-80$ & Con & Eph & 535 \\
\hline 64 & 06378300 & Lodgepole Creek near Hampshire & $1978-80$ & Con & Eph & 354 \\
\hline 65 & 06379600 & Box Creek near Bill & $1956-58,1961-80$ & $\mathbf{P R}$ & Eph & 112 \\
\hline 66 & 06382200 & Pritchard Draw near Lance Creek & $1964-80$ & PR & Eph & 5.1 \\
\hline 67 & 06386000 & Lance Creek near Riverview & $1949-54,1957-80$ & Con & Eph & 2,070 \\
\hline 68 & 06386500 & Cheyenne River near Riverview & $1949-73$ & Con & Eph & 5,270 \\
\hline 69 & 06387500 & Turner Creek near Osage & $1959-80$ & PR & Eph & 47.8 \\
\hline 70 & 06388800 & $\begin{array}{l}\text { Blacktail Creek tributary near } \\
\text { Newcastle }\end{array}$ & $1960-80$ & $\mathbf{P R}$ & Eph & .25 \\
\hline 71 & 06392900 & $\begin{array}{l}\text { Beaver Creek at Mallo Camp near } \\
\text { Four Corners }\end{array}$ & $1975-80$ & Con & Per & 10.3 \\
\hline 72 & 06392950 & $\begin{array}{l}\text { Stockade Beaver Creek near } \\
\text { Newcastle }\end{array}$ & $1975-80$ & Con & Per & 107 \\
\hline $\begin{array}{l}73 \\
74\end{array}$ & $\begin{array}{l}06394000 \\
06395000\end{array}$ & $\begin{array}{l}\text { Beaver Creek near Newcastle } \\
\text { Cheyenne River at Edgemont, S.D. }\end{array}$ & $\begin{array}{l}1945-80 \\
1930-32,1947-80\end{array}$ & $\begin{array}{l}\text { Con } \\
\text { Con }\end{array}$ & $\begin{array}{l}\text { Per } \\
\text { Int }\end{array}$ & $\begin{array}{l}1,320 \\
7,143\end{array}$ \\
\hline
\end{tabular}


Table 1.--Streamflow stations and sites included in assessment of low flows--Continued

\begin{tabular}{|c|c|c|c|c|c|c|}
\hline $\begin{array}{l}\text { ID } \\
\text { ne. }\end{array}$ & $\begin{array}{l}\text { Permanent } \\
\text { station } \\
\text { number }\end{array}$ & Station name & $\begin{array}{l}\text { Post-1929 } \\
\text { period } \\
\text { of } \\
\text { recerd }\end{array}$ & $\begin{array}{l}\text { Record } \\
\text { type }\end{array}$ & $\begin{array}{l}\text { Stream } \\
\text { type }\end{array}$ & $\begin{array}{l}\text { Drainage } \\
\text { area } \\
\text { (square } \\
\text { miles) } \\
\end{array}$ \\
\hline \multicolumn{7}{|c|}{ Belle Fourche River Basin } \\
\hline 75 & 06409000 & $\begin{array}{l}\text { Castle Creek above Deerfield } \\
\text { Reservoir near Hill City, S.D. }\end{array}$ & $1949-80$ & Con & Per & 83 \\
\hline 76 & 06425720 & $\begin{array}{l}\text { Belle Fourche River below } \\
\text { Rattlesnake Creek near Piney }\end{array}$ & $1976-80$ & Con & Int & 495 \\
\hline 77 & 06425780 & $\begin{array}{l}\text { Belle Fourche River above Dry } \\
\text { Creek near Piney }\end{array}$ & $1976-80$ & Con & Int & 594 \\
\hline 78 & 06425900 & Caballo Creek at mouth near Piney & $1978-80$ & Con & Eph & 260 \\
\hline 79 & 06425950 & Raven Creek near Moorcroft & $1978-80$ & Con & Eph & 76 \\
\hline 80 & 06426000 & Belle Fourche River near Moorcroft & $1930-32$ & Con & Int & 1,380 \\
\hline 81 & 06426400 & Donkey Creek near Moorcroft & $1978-80$ & Con & Int & 246 \\
\hline 82 & 06426500 & $\begin{array}{l}\text { Be1le Fourche River below } \\
\text { Moorcroft }\end{array}$ & $1944-70,1976-80$ & Con & Int & 1,670 \\
\hline 83 & -- & Wind Creek near Moorcroft & 1975 & Misc & Eph & -- \\
\hline 84 & -- & Mule Creek near Moorcroft & 1975 & Misc & Eph & -- \\
\hline 85 & 06428000 & Belle Fourche River at Hulett & $1930-32,1939-51$ & Con & Per & 2,800 \\
\hline 86 & 06428100 & $\begin{array}{l}\text { Belle Fourche tributary no. } 2 \\
\text { near Hulett }\end{array}$ & $1962-80$ & PR & Per & 10.2 \\
\hline 87 & 06428500 & $\begin{array}{l}\text { Belle Fourche River at Wyoming- } \\
\text { South Dakota State line }\end{array}$ & $1947-80$ & Con & Int & 3,280 \\
\hline $\begin{array}{l}88 \\
89\end{array}$ & $\begin{array}{l}06429300 \\
06429500\end{array}$ & $\begin{array}{l}\text { Ogden Creek near Sundance } \\
\text { Cold Spring } 8 \text { Creek at Buckhorn }\end{array}$ & $\begin{array}{l}1962-80 \\
1975-80\end{array}$ & $\begin{array}{l}\text { PR } \\
\text { Con }\end{array}$ & $\begin{array}{l}\text { Int } \\
\text { Per }\end{array}$ & $\begin{array}{l}8.42 \\
19.0\end{array}$ \\
\hline 90 & 06430500 & $\begin{array}{l}\text { Redwater Creek at Wyoming-South } \\
\text { Dakota State line }\end{array}$ & $1930-31,1955-80$ & Con & Per & 471 \\
\hline \multicolumn{7}{|c|}{ Niobrara Riyer Basin } \\
\hline 91 & 06454000 & $\begin{array}{l}\text { Niobrara River at Wyoming- } \\
\text { Nebraska State line }\end{array}$ & $1956-80$ & Con & Per & 450 \\
\hline \multicolumn{7}{|c|}{ North Platte River Bas in } \\
\hline 92 & 06641400 & Bear Springs Creek near Alcova & $1960-80$ & PR & Eph & 9.48 \\
\hline 93 & 06642000 & North Platte River at Alcova & $1961-80$ & Con & Per & 10,812 \\
\hline 94 & 06643000 & Bates Creek near Alcova & $1936-54,1957-61$ & Con & Int & 393 \\
\hline 95 & 06643300 & Coal Creek near Goose Egg & $1960-80$ & PR & Int & 5.39 \\
\hline 96 & 06644000 & $\begin{array}{l}\text { Poison Spider Creek near Goose } \\
\text { Egg }\end{array}$ & $1951-56$ & Con & Per & 301 \\
\hline 97 & 06644120 & $\begin{array}{l}\text { Middle Fork Casper Creek near } \\
\text { Casper }\end{array}$ & $1968-75$ & PR & Int & -- \\
\hline 98 & 06644500 & Casper Creek at Casper & $1947-56$ & Con & Int & 668 \\
\hline 99 & 06644840 & $\begin{array}{l}\text { McKenzie Draw tributary near } \\
\text { Casper }\end{array}$ & $1965-80$ & PR & Per & 2.02 \\
\hline 100 & 06646300 & $\begin{array}{l}\text { Little Deer Creek below East Cart } \\
\text { Creek near Glenrock }\end{array}$ & $1975-76$ & Con & Int & 7.48 \\
\hline
\end{tabular}


Table 1.--Streamf low stations and sites included in assessment of lor flors--Cont inued

\begin{tabular}{|c|c|c|c|c|c|c|}
\hline $\begin{array}{l}\text { ID } \\
\text { ne. }\end{array}$ & $\begin{array}{l}\text { Permanent } \\
\text { station } \\
\text { number }\end{array}$ & Station name & $\begin{array}{l}\text { Post-1929 } \\
\text { period } \\
\text { of } \\
\text { record }\end{array}$ & $\begin{array}{l}\text { Record } \\
\text { type }\end{array}$ & $\begin{array}{l}\text { Stream } \\
\text { type }\end{array}$ & $\begin{array}{c}\text { Drainage } \\
\text { area } \\
\text { (square } \\
\text { miles) }\end{array}$ \\
\hline \multicolumn{7}{|c|}{ Nerth Platte Biyer Basin--Continued } \\
\hline 101 & 06646500 & Deer Creek at Glenrock & $1930-33,1936-60$ & Con & Int & 212 \\
\hline L/ & 06646600 & $\begin{array}{l}\text { Deer Creek below Miller Wasteway } \\
\text { near Glenrock }\end{array}$ & $1962-80$ & Con & Int & 213 \\
\hline 102 & 06646700 & $\begin{array}{l}\text { East Fork Dry Creek tributary } \\
\text { near Glenrock }\end{array}$ & $1961-80$ & Con & Eph & 2.60 \\
\hline $\begin{array}{l}103 \\
104\end{array}$ & $\begin{array}{l}06646780 \\
06646800\end{array}$ & $\begin{array}{l}\text { Sand Creek near Glenrock } \\
\text { North Platte River near Glenrock }\end{array}$ & $\begin{array}{l}1978-80 \\
1961-80\end{array}$ & $\begin{array}{l}\text { Con } \\
\text { Con }\end{array}$ & $\begin{array}{l}\text { Eph } \\
\text { Per }\end{array}$ & $\begin{array}{r}79.9 \\
13,538\end{array}$ \\
\hline 105 & 06647500 & Box Elder Creek at Boxelder & $\begin{array}{l}1947-51,1962-67, \\
1972-80\end{array}$ & Con & Per & 63.0 \\
\hline 106 & 06647900 & $\begin{array}{l}\text { Little Box Blder Creek at Little } \\
\text { Box Cave near Careyhurst }\end{array}$ & $1975-80$ & Con & Eph & 8.47 \\
\hline $\begin{array}{l}107 \\
108\end{array}$ & $\begin{array}{l}06648000 \\
06648720\end{array}$ & $\begin{array}{l}\text { Box Elder Creek near Careyhurst } \\
\text { Frank Draw tributary near Orpha }\end{array}$ & $\begin{array}{l}1930-32,1936-69 \\
1965-73\end{array}$ & $\begin{array}{l}\text { Con } \\
\text { PR }\end{array}$ & $\begin{array}{l}\text { Int } \\
\text { Eph }\end{array}$ & $\begin{array}{l}202 \\
.79\end{array}$ \\
\hline 109 & 06648780 & Sage Creek tributary near Orpha & $1965-80$ & $\mathbf{P R}$ & Eph & 1.38 \\
\hline $\begin{array}{l}110 \\
111\end{array}$ & $\begin{array}{l}06649000 \\
06649500\end{array}$ & $\begin{array}{l}\text { La Prele Creek near Douglas } \\
\text { La Prele Creek near Orpha }\end{array}$ & $\begin{array}{l}1930-71 \\
1933,1936-70\end{array}$ & $\begin{array}{l}\text { Con } \\
\text { Con }\end{array}$ & $\begin{array}{l}\text { Per } \\
\text { Int }\end{array}$ & $\begin{array}{l}135 \\
177\end{array}$ \\
\hline 112 & 06649900 & $\begin{array}{l}\text { Nortb Platte River tributary } \\
\text { near Douglas }\end{array}$ & $1961-80$ & PR & Eph & 8.53 \\
\hline 113 & 06650500 & Wagonhound Creek near La Bonte & $1940-69$ & Con & Int & 112 \\
\hline 114 & 06651500 & La Bonte Creek near La Bonte & $1930-32,1936-69$ & Con & Int & 287 \\
\hline $\begin{array}{l}115 \\
116\end{array}$ & $\begin{array}{l}06651800 \\
06652000\end{array}$ & $\begin{array}{l}\text { Sand Creek near Orin } \\
\text { North Platte River at Orin }\end{array}$ & $\begin{array}{l}1955,1961-80 \\
1961-80\end{array}$ & $\begin{array}{l}\text { PR } \\
\text { Con }\end{array}$ & $\begin{array}{l}\text { Int } \\
\text { Per }\end{array}$ & 14,888 \\
\hline 117 & 06652400 & Watson Draw near Lost Springs & $1960-80$ & PR & Eph & 6.95 \\
\hline 118 & -- & Elkhorn Creek near Glendo & 1975 & Misc & Eph & -- \\
\hline 119 & -- & Cottonwood Creek near Glendo & 1975 & Misc & Eph & -- \\
\hline 120 & -- & Whiskey Gulch near Glendo & 1975 & Misc & Eph & -- \\
\hline 121 & 06652800 & $\begin{array}{l}\text { North Platte River below Glendo } \\
\text { Reservoir }\end{array}$ & $1958-80$ & Con & Per & 15,548 \\
\hline 122 & 06653000 & Horseshoe Creek near Esterbrook & $1947-51$ & Con & Int & 45.5 \\
\hline 123 & 06653300 & Horseshoe Creek near Cassa & $1962-68$ & Con & Int & 180 \\
\hline 124 & 06653500 & Horseshoe Creek near Glendo & $1936-70$ & Con & Int & 211 \\
\hline 125 & 06654550 & Cottonwood Creek near Binford & 1974 & Con & Int & 61.0 \\
\hline 126 & 06655000 & Cottonwood Creek at Wendover & $\begin{array}{l}1930-32,1936-42 \\
1947-55,1974\end{array}$ & Con & Per & 196 \\
\hline 127 & 06656000 & $\begin{array}{l}\text { North Platte River below } \\
\text { Guernsey Reservoir }\end{array}$ & $1930-80$ & Con & Per & 16,237 \\
\hline 128 & 06671000 & Rawhide Creek near Lingle & $1930-71$ & Con & Per & 522 \\
\hline
\end{tabular}

$1 /$ Low-flow records for these stations are considered equivalent and were combined as one station for frequency analysis. 


\section{Minimum Observed Flows}

The minimum flow of record is an important low-flow characteristic (Riggs, 1980, p. 722). The minimum instantaneous discharge during the period of record at each streamflow station and observed no flow at each miscellaneous-observation site are shown on plate 1. The minimum flow is zero at almost all stations and sites except those in the mountain areas, a few in the foothills adjacent to the mountains, and those on main stems of principal streams on the plains. A notable exception is Salt Creek near Sussex (ID no. 30), where the minimum observed flow is 5 cubic feet per second, even though the station is far from the mountains. Salt Creek has perennial base flow because of water discharged to the stream from a nearby oil field. Prior to about the 1930's Salt Creek was an ephemeral plains stream (Bille, 1978). Because the record for Salt Creek is considered to be artificial, it was not used in the statistical analysis of low flows.

\section{Flow-Duration Curves}

Flow-duration curves are cumulative frequency curves that show the percent of time during which specified discharges were equalled or exceeded at a streamflow station during a given period of record. They provide "a convenient means for studying the flow characteristics of a stream and for comparing one basin with another" (Searcy, 1959, p. 1-2). Riggs (1972, p. 15) states: "The lower end of the duration curve is an expression of the low-flow characteristics of a stream, but it provides less information than a low-flow frequency curve, because the duration curve applies to the period of record rather than to a year."

Although flow-duration curves are not the principal analytical technique used in this study, they are useful to illustrate the substantial differences in flow types in streams of northeastern Wyoming. Examples showing the differences are shown in figure 3. The curve for the mountain stream (which is perennial) is flat on the lower end, indicating the existence of substantial storage of surface or ground water in the basin for sustaining the flow. In contrast, the lower ends of the two curves for the plains stream (which is perennial, but goes dry during some years) are very steep, indicating negligible storage and no flow about 4 percent of the time during the period, 1962-80. The curves for the plains stream also demonstrate the differences in low-flow characteristics when the period of record does not contain a representative sample of low flows. Low flows during the selected shorter period (1962-82), which does not include a drought, are considerably larger than low flows during the cormlete period of record (1931-33, 1935-80).

Curves for other streams in the area are similar to the examples in figure 3. Tables of flow-duration data for most stations on perennial or intermittent streams may be requested from the Cheyenne, Wyo. office of the Geological Survey. Generally, flow-duration data for ephemeral streams have little use because of the extended periods of no flow in such streams. 


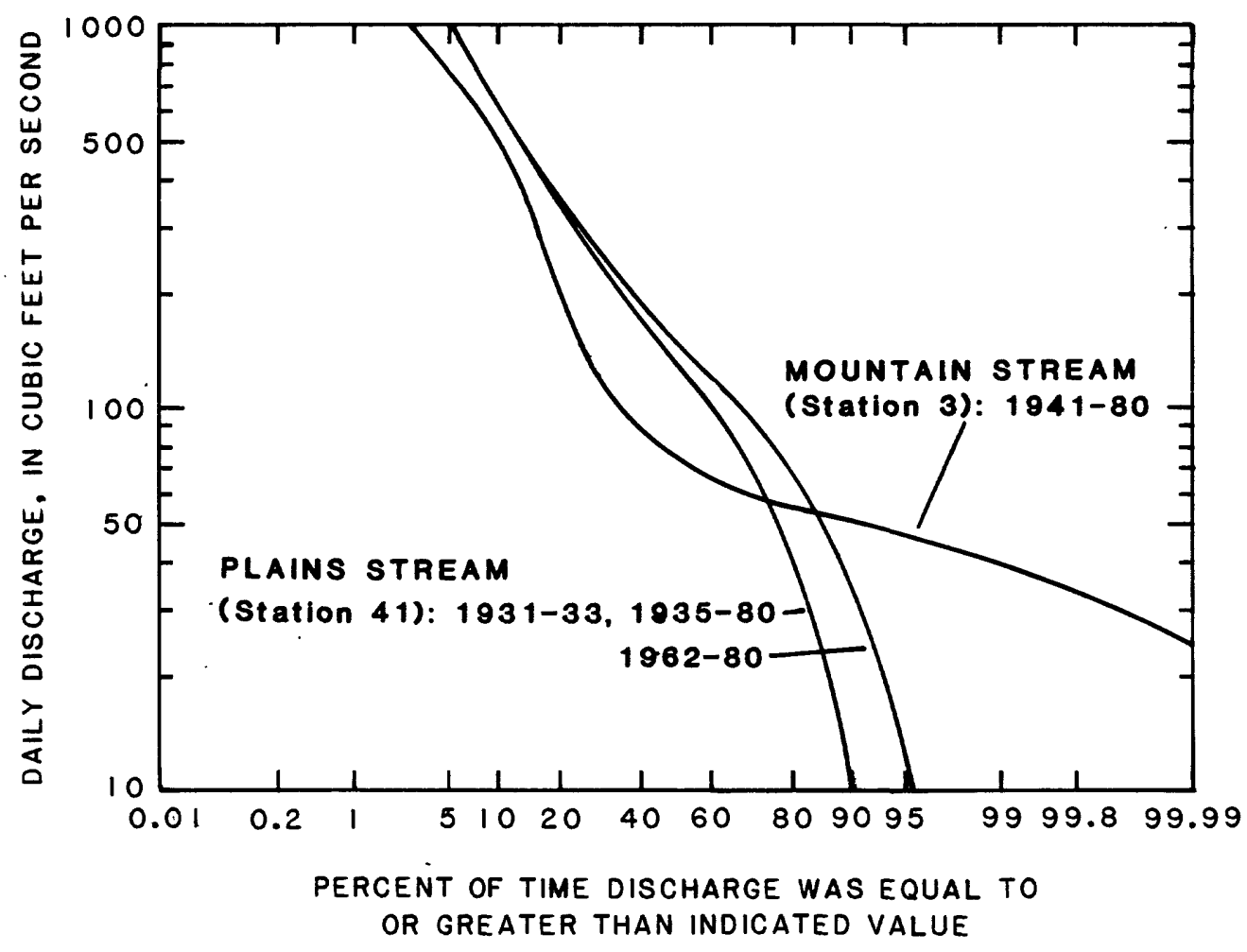

Figure 3.--Flow-duration curves for a mountain stream and a plains stream in northeastern Wyoming. (Station names are given in table 1.) 
Frequency Analysis

The principal analytical procedure used in this study was the statistical analysis of the magnitude and frequency of low flows at streamflow stations. This "standard" procedure is described by Riggs (1972). Time and resources available for this study did not permit evaluation of other techniques described by Riggs (1972), such as analysis of base-flow recession curves. Although the results of frequency analysis have limitations, they are considered to be adequate as general indicators of low-flow characteristics of streams. Frequency analysis was applied to records for perennial and selected intermittent streams-mainly mountain and main-stem plains streams. Because ephemeral streams in the plains are dry most of the time, the frequency of periods of no flow was determined for ephemeral streams.

\section{Seven-Day Low Flows}

Minimum seven-day average flows for selected recurrence intervals were calculated from streamflow records at stations on perennial mountain streams and on some perennial and intermittent plains streams. The calculations were made for stations on 42 perennial streams and 13 intermittent streams, at which records had been collected for at least 10 years and included a drought. Most stations installed after 1960 were excluded because the records do not include the droughts of the 1930's or 1950's.

Calculations of 7-day low flow were made for some stations where the flow is affected by human activities. Same larger streams, such as Clear Creek, the upstream reaches of the Powder River, and the North Platte River, convey flows that originate in the mountains through or far onto the plains. Flow in same mountain streams is used and reused as it is conveyed to the plains; consequently, low flows in the plains reaches of these streams consist mainly of the seepage water and return flows. Generally, this water use has been consistent enough that the low-flow data can be analyzed statistically in the same manner as data for undisturbed streams.

Low-flow characteristics of regulated streams apply only so long as the pattern of regulation is unchanged (Riggs, 1980, p. 729). The North Platte River is completely regulated; its flows are determined by release rules that generally are implemented based on variation in inflow. Because the pattern of regulation has been more or less the same throughout the period of record used in the analysis, low-flow characteristics were determined for stations on the North Platte. Low-flow characteristics were not determined for stations downstream from small dams on streams in the mountains.

For selected stations the frequency of minimum average-annual discharge for 7 consecutive days was calculated, based on the climatic year (AprilMarch). The results are listed in table 2. The 7-day low flow commonly is used as the principal low-flow indicator; frequency values for other periods, such as 1, 3, 14, 30, and 60 consecutive days, were calculated but are not included in this report. This information is available from the Geological Survey office in Cheyenne, Wyo. 
Table 2.-Frequency distribution of minimum average 7-day flows at selected streamflow stations with sufficient records to include a drought, in northeastern Wyaming and adjacent parts of Montana and South Dakota.

[ID no.: Station identification number used in this report (see table 1); data in table: discharge, in cubic feet per second]

\begin{tabular}{rcccccccc}
\hline \multicolumn{7}{c}{ Probability that annual minimum discharge will not } \\
\multicolumn{7}{c}{} & \multicolumn{7}{c}{ exceed the indicated value (percent) } & \\
ID & 5 & 10 & 20 & 50 & 80 & 90 & 99 \\
\cline { 2 - 8 } no. & 20 & 10 & 5 & 2 & 1.25 & 1.11 & 1.01 \\
\hline 1 & 36 & 39 & 43 & 50 & 58 & 62 & 72 \\
2 & 7.3 & 7.9 & 8.6 & 10 & 12 & 13 & 17 \\
3 & 39 & 40 & 42 & 45 & 49 & 51 & 57 \\
4 & .93 & 1.0 & 1.2 & 1.5 & 1.8 & 2.0 & 2.5 \\
5 & 2.7 & 3.0 & 3.3 & 3.9 & 4.4 & 4.6 & 5.0 \\
7 & 1.1 & 1.3 & 1.5 & 2.1 & 2.8 & 3.2 & 4.5 \\
8 & 2.9 & 3.7 & 4.7 & 7.1 & 10 & 12 & 16 \\
9 & 5.2 & 5.5 & 5.9 & 6.9 & 8.0 & 8.6 & 10 \\
10 & 8.4 & 11 & 16 & 27 & 43 & 53 & 79 \\
12 & 6.4 & 13 & 27 & 70 & 130 & 160 & 210 \\
15 & 16 & 18 & 20 & 25 & 30 & 34 & 43 \\
17 & .79 & .92 & 1.1 & 1.5 & 1.9 & 2.2 & 2.7 \\
18 & 9.2 & 10 & 12 & 15 & 17 & 18 & 20 \\
20 & .09 & .15 & .29 & 1.0 & 3.6 & 6.8 & 32 \\
24 & .23 & .52 & 1.1 & 3.1 & 4.8 & 5.2 & 5.5 \\
32 & 4.8 & 5.1 & 5.6 & 8.0 & 15 & 23 & 89 \\
35 & 1.2 & 1.3 & 1.6 & 2.3 & 3.2 & 3.8 & 5.6 \\
36 & 0 & 0 & .45 & 1.5 & 3.0 & 3.8 & 5.6 \\
37 & 2.4 & 2.8 & 3.5 & 5.0 & 6.8 & 7.8 & 10 \\
39 & 0 & 0 & 0 & 0 & 5.5 & 14 & 47 \\
41 & 0 & 0 & 0 & 0 & 23 & 35 & 90 \\
43 & 1.4 & 1.7 & 2.2 & 3.2 & 4.2 & 4.7 & 5.5 \\
44 & .20 & .36 & .71 & 2.4 & 6.9 & 12 & 33 \\
46 & .96 & 1.3 & 1.9 & 3.1 & 4.4 & 4.9 & 5.7 \\
47 & 3.6 & 3.9 & 4.3 & 5.1 & 6.0 & 6.5 & 7.9 \\
48 & 4.4 & 5.0 & 6.0 & 8.4 & 12 & 14 & 22 \\
49 & 1.4 & 2.5 & 4.6 & 11 & 19 & 23 & 30 \\
50 & .10 & .14 & .24 & .52 & .81 & .90 & .99 \\
51 & 0 & .38 & 2.6 & 16 & 45 & 63 & 99 \\
57 & 0 & 0 & .03 & .55 & 1.8 & 2.5 & 3.9 \\
58 & 0 & 0 & 0 & 0 & .02 & .11 & .39 \\
73 & 0 & 0 & 0 & .21 & 1.0 & 1.8 & 6.2 \\
75 & 3.2 & 3.8 & 4.6 & 6.2 & 7.6 & 8.3 & 9.6 \\
82 & 0 & 0 & 0 & 0 & 0 & .05 & 3.1 \\
85 & 0 & 0 & 0 & .85 & 2.6 & 4.3 & 12 \\
87 & 0 & 0 & 0 & 3.5 & 12 & 14 & 16 \\
90 & 4.2 & 5.0 & 6.3 & 9.9 & 16 & 22 & 43 \\
91 & .77 & .95 & 1.2 & 1.7 & 2.3 & 2.6 & 3.3 \\
93 & 180 & 227 & 294 & 448 & 624 & 718 & 928 \\
94 & 0 & 0 & 0 & .05 & .14 & .20 & .47 \\
101 & 0 & 0 & 0 & .16 & .30 & .40 & .76 \\
& 0 & 0 & 0 & .06 & .38 & .84 & 4.4
\end{tabular}


Table 2. - Frequency distribution of minimum average 7-day flows at selected streamflow stations with sufficient records to include a drought--Continued

\begin{tabular}{|c|c|c|c|c|c|c|c|}
\hline \multirow[b]{3}{*}{ ID } & \multicolumn{7}{|c|}{$\begin{array}{l}\text { Probability that annual minimum discharge will not } \\
\text { exceed the indicated value (percent) }\end{array}$} \\
\hline & 5 & 10 & 20 & 50 & 80 & $90^{\circ}$ & 99 \\
\hline & \multicolumn{7}{|c|}{ Recurrence interval (years) } \\
\hline no. & 20 & 10 & 5 & 2 & 1.25 & 1.11 & 1.01 \\
\hline$\overline{104}$ & 355 & 405 & 466 & 574 & 658 & 690 & 736 \\
\hline 105 & 0 & 0 & 0 & .09 & .28 & .47 & 1.5 \\
\hline 107 & .08 & .13 & .20 & .36 & .59 & .76 & 1.2 \\
\hline 110 & 0 & .01 & .31 & 1.6 & 3.0 & 3.5 & 3.9 \\
\hline 111 & 0 & 0 & 0 & .09 & .67 & 1.7 & 22 \\
\hline 113 & 0 & 0 & 0 & .08 & .21 & .31 & .7 \\
\hline 114 & 0 & 0 & 0 & 1.8 & 3.5 & 3.9 & 4.1 \\
\hline 116 & 403 & 451 & 509 & 619 & 718 & 764 & 850 \\
\hline 121 & .93 & 1.1 & 1.3 & 2.0 & 3.3 & 4.4 & 9.9 \\
\hline 124 & .01 & .24 & .83 & 2.3 & 3.4 & 3.7 & 3.8 \\
\hline 126 & .70 & .88 & 1.1 & 1.8 & 2.5 & 3.0 & 4.1 \\
\hline 127 & .78 & 1.8 & 4.8 & 29 & 155 & 358 & 2,310 \\
\hline 128 & .35 & .90 & 2.0 & 5.7 & 12 & 16 & 25 \\
\hline
\end{tabular}

The probabilities that the 7-day low flow during any given year will be less than specified values are given in table 2. For example, at station 10 there is a probability of 0.10 (10 percent chance) that the minimum average 7day flow in any year will be less than 11 cubic feet per second, and a probability of 0.90 ( 90 percent chance) that the flow will be less than 53 cubic feet per second. Low flows with recurrence intervals greater than 20 years (probabilities less than 5 percent) are not listed in table 2.

Calculated values of frequency distributions typically are uneven, particularly if the period of record is relatively short. For each station the log Pearson Type III frequency distribution was fitted to the data using the mean, standard deviation, and skew coefficient calculated from the data. The data in table 2 are log Pearson Type III results. Typical log Pearson Type III frequency curves for $1,3,7,14,30$, and 60-day low flows are shown in figure 4. Like the flow-duration curves (fig. 3), the curves in figure 4 illustrate the difference between streams that always flow and streams that occasionally go dry.

From the range of recurrence intervals for the 7-day low flow listed in table 2, the 7-day 10-year low flow $\left(7 Q_{10}\right)$ was selected for further regional analysis. The $7 Q_{10}$ commonly is cited as a standard to which laws and regulations apply (Riggs, 1980, p. 721). It also is used to design water-supply projects (Chang and Boyer, 1977, p. 997) and to facilitate water management (Chiang and Johnson, 1976). The 7-day low flow will be less than the 7Q ${ }_{10}$ at intervals averaging 10 years in length; the probability is 0.10 (10 percent chance) that the 7-day low flow in any one year will be less than the $70_{10}$. The areal distribution of the $7 Q_{10}$ in mountain and main-stem plains streams ${ }^{1}$ s shown on plate 1 . 


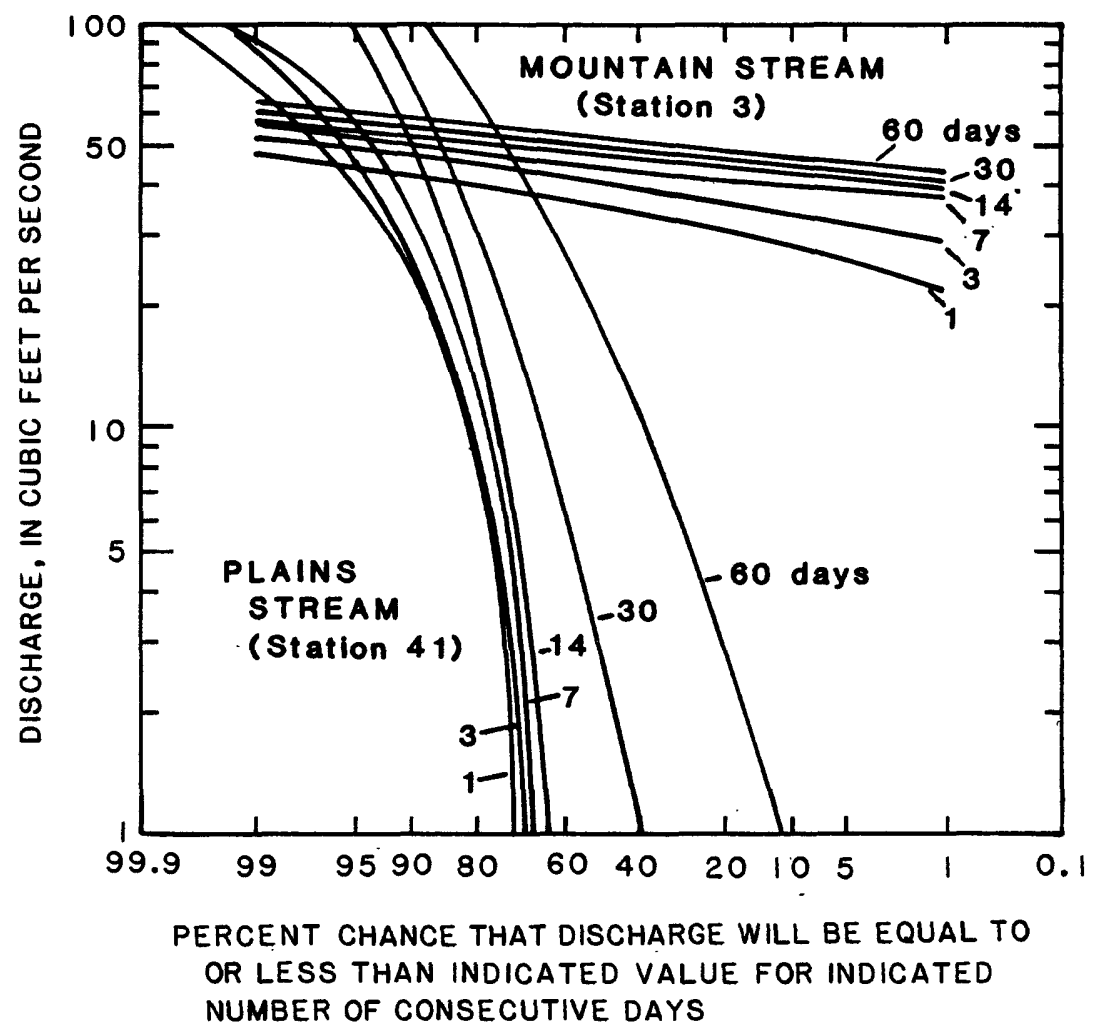

Figure 4.--Low-flow frequency curves for a mountain stream and a plains stream in northeastern Wyoming. (Station names are given in table 1.) 


\section{No-Flow Periods}

Most streams that originate in the foothills and plains, including the semiarid north slope of the Laramie Mountains, have no flow during part of every year. In fact, many of these streams are ephemeral. Therefore, standard methods of frequency analysis could not be used. Instead, records for stations on plains and foothills streams were analyzed for the frequency of the annual maximum number of consecutive days of no flow. Frequency analysis of no-flow days provides an indication of the likelihood of the occurrence of extended periods of no flow or drought.

Due to the lack of information for plains streams, short-term records were used for the determination of the frequency of no-flow periods. The only plains-stream records available prior to about 1972 were on the large streams. Generally, the records do not include the droughts of the 1930's or 1950's, and were used only if they contained periods of no flow.

Frequency data for periods of no flow in plains and foothill streams are listed in table 3. Frequencies were not computed for 5 stations that had less than 10 years of record. Data for some of the stations used in the analysis of 7-day low flows also were used in the analysis of no-flow days. The table includes the maximum observed number of consecutive days of no flow during the period of record for all stations and the 7-day 10-year low flow for stations with 10 or more years of record, regardless of whether the period of record includes a major drought. Smoothed curves for five stations are shown in figure 5. All five stations have long periods of record that include a major drought.

\section{LOW-FLOW CHARACTERISTICS AT UNGAGED SITES}

Although the scope of this study was limited mainly to the general summary of low-flow characteristics described in the preceding sections, the possibility of applying regression techniques to the data was investigated. This was done because of the potential usefulness of equations for predicting low-flow characteristics at ungaged sites. Geologic factors, which have an important effect on low flows but are difficult to quantify as independent variables, were not investigated. Simple regression for $7 Q_{10}$ at mountain stations and interpolative graphs for selected main-stem streams are reported here.

Most of the streams in the study area are ephemeral; however, equations for predicting periods of no-flow days or other charateristics at sites on plains streams could not be developed. Although the maximum number of no-flow days was found to be correlated with average annual discharge, average annual discharge itself would have to be estimated from regression equations, such as those of Lowham (1976). Therefore, no-flow periods for ephemeral plains streams can be estimated only by areal interpolation of the data shown on plate 1 or listed in table 3 . 
Table 3.- Seven-day 10-year low flows and frequency of no flow for selected periods of consecutive days, at selected stations on plains and foothills streams in northeastern wyoming and adjacent parts of Montana and South Dakota

[ID no.: Station identification number used in this report (see table 1); 70 10 : 7-day 10-year low flow, in cubic feet per second; max: maximum annual number of consecutive days of no flow recorded at station; <, less than; >, greater than]

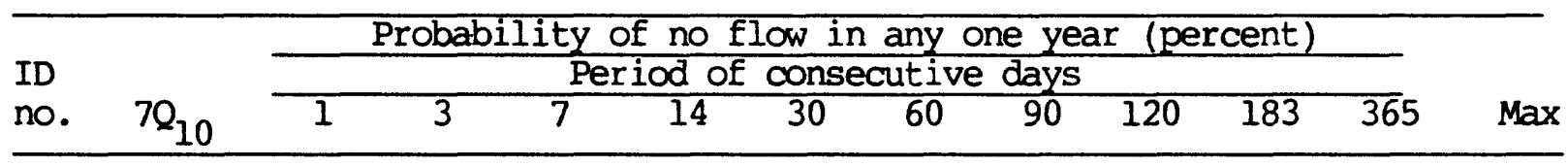

$16 \quad(1) \quad 12$

$\begin{array}{llllll}20 & 0.15 & 3 & <1 & 1\end{array}$

$\begin{array}{llllllllll}24 & .52 & 14 & 9 & 9 & 9 & 5 & 5 & <1 & 79\end{array}$

29 (1) 344

$\begin{array}{lllllllll}33 & 0 & >99 & 88 & 88 & 63 & 25 & <1 & 294\end{array}$

$\begin{array}{lllllllll}36 & 0 & 17 & 17 & 11 & <1 & 11\end{array}$

$\begin{array}{lllllllllll}39 & 0 & 61 & 61 & 57 & 48 & 43 & 30 & 4 & <1 & 139\end{array}$

$\begin{array}{llllllllll}41 & 0 & 71 & 69 & 65 & 52 & 21 & 4 & <1 & 91\end{array}$

$\begin{array}{lllll}44 & .36 & 2 & <1 & \end{array}$

$\begin{array}{llllllll}50 & .14 & 10 & 8 & 5 & 2 & <1 & 26\end{array}$

$\begin{array}{llllllll}51 & .38 & 15 & 15 & 7 & 7 & <1 & 26\end{array}$

54 (1) 21

$\begin{array}{llllllllll}57 & 0 & 37 & 32 & 16 & 16 & 5 & <1 & 88\end{array}$

$\begin{array}{lllllllllllll}58 & 0 & 83 & 83 & 77 & 60 & 43 & 34 & 23 & 20 & 11 & <1 & 286\end{array}$

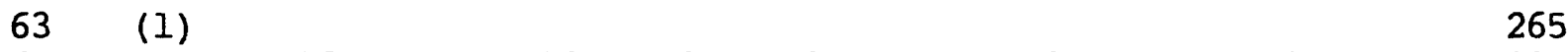

$\begin{array}{lllllllllllll}67 & 0 & 96 & 96 & 96 & 93 & 86 & 57 & 46 & 32 & 2 & <1 & 330\end{array}$

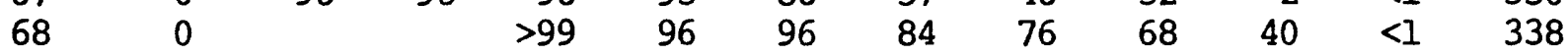

$\begin{array}{llllllllllll}73 & 0 & 37 & 31 & 26 & 23 & 20 & 6 & 3 & <1 & & 174\end{array}$

$\begin{array}{llllllllllll}74 & 0 & 70 & 64 & 61 & 39 & 21 & 9 & 6 & 6 & <1 & 239\end{array}$

76 (1) 235

$\begin{array}{llll}77 & \text { (1) } & 152\end{array}$

$\begin{array}{lllllllllllll}82 & 0 & 90 & 90 & 87 & 87 & 77 & 63 & 43 & 30 & 20 & <1 & 309\end{array}$

$\begin{array}{lllllllllll}85 & 0 & 21 & 21 & 21 & 14 & 14 & 7 & <1 & 72\end{array}$

$\begin{array}{llllllllllll}87 & 0 & 36 & 36 & 36 & 33 & 27 & 18 & 6 & 6 & <1 & 224\end{array}$

$\begin{array}{llllllllll}94 & 0 & 59 & 55 & 50 & 41 & 23 & 5 & <1 & 109\end{array}$

$\begin{array}{llllllll}98 & 0 & 22 & 22 & 22 & 11 & <1 & 114\end{array}$

$\begin{array}{llllllllll}101 & 0 & 41 & 39 & 35 & 26 & 11 & 4 & <1 & 75\end{array}$

$\begin{array}{llllllll}105 & 0 & 47 & 41 & 35 & 6 & <1 & 28\end{array}$

$\begin{array}{lllllll}107 & .13 & 14 & 6 & 3 & <1 & 21\end{array}$

$\begin{array}{llllllll}110 & .01 & 13 & 12 & 10 & 2 & <1 & 45\end{array}$

$\begin{array}{llllllllllll}111 & 0 & 53 & 39 & 39 & 36 & 19 & 11 & 11 & 3 & <1 & 138\end{array}$

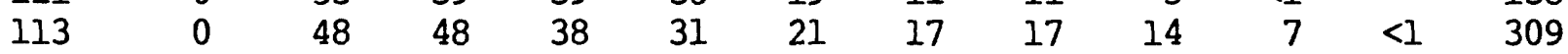

$\begin{array}{llllllllllr}114 & 0 & 26 & 26 & 23 & 20 & 14 & 9 & <1 & 85\end{array}$

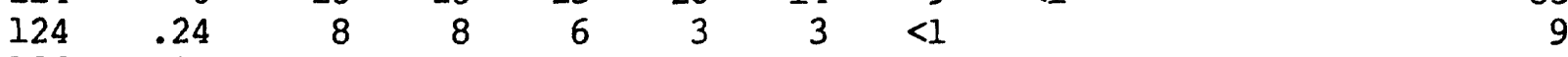

$\begin{array}{lllll}126 & .88 & 5 & <1 & 2\end{array}$

$\frac{128 \quad .90}{1}$ Not calculated. 


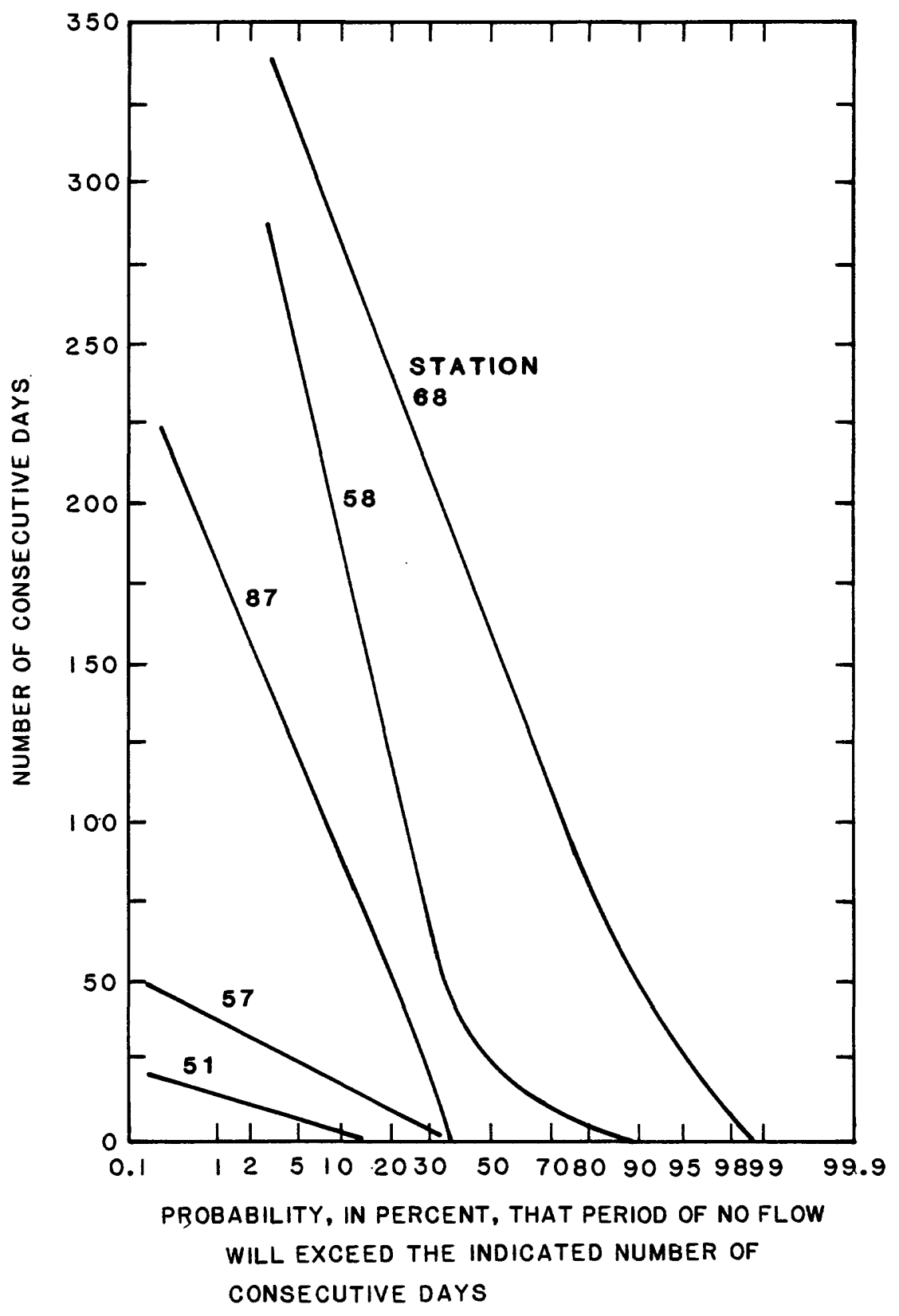

Figure 5.--Frequency curves for maximum number of consecutive days of no flow at five stations on plains streams in northeastern Wyoming and adjacent parts of Montana. (Station names are given in table 1.) 


\section{Mountain Streams}

The $7 Q_{10}$ for streams in the Bighorn Mountains (see plate 1) was correlated with drainage area. The regression equation is shown graphically in figure 6. It was determined using 19 stations; the correlation coefficient is 0.71 and the standard error is 64 percent. The equation may be used to estimate $7 Q_{10}$ at ungaged sites in the Bighorns only, but the applicable area is only a sinall part of the study area.

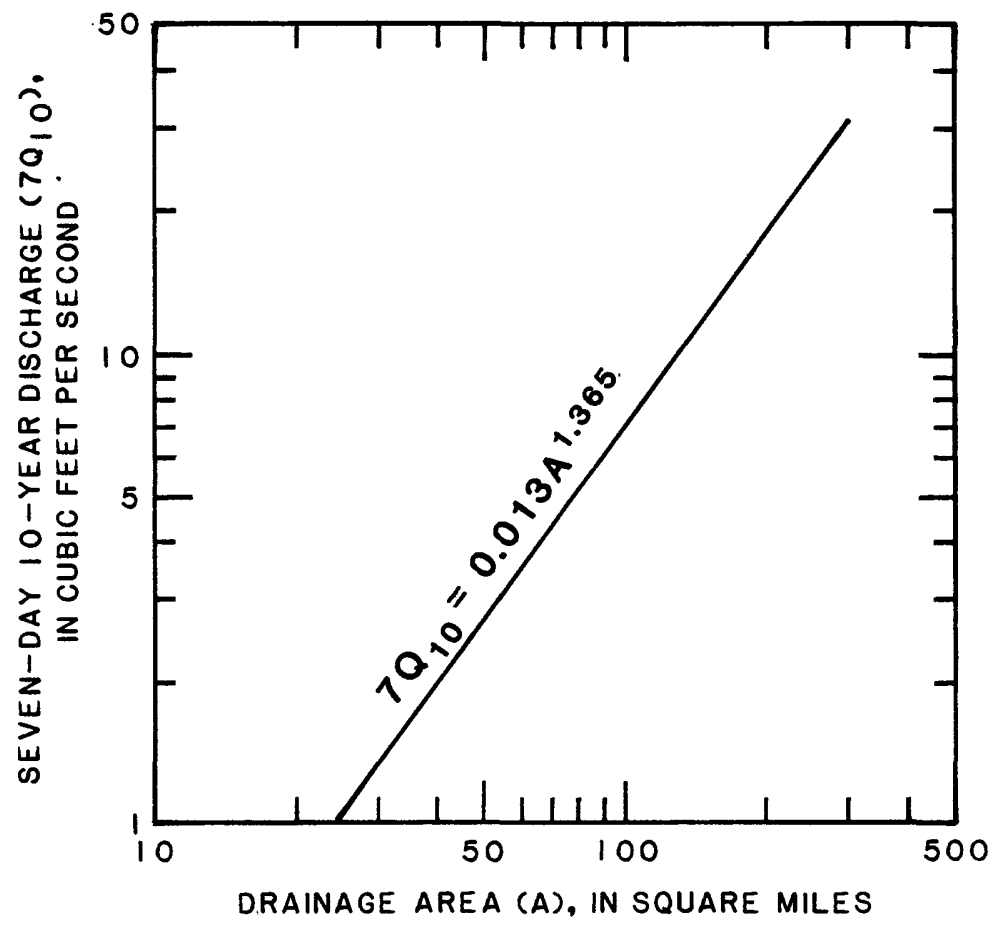

Figure 6.--Relation of 7-day 10-year low flow to drainage area, for streams in the Bighorn Mountains and Black Hills in Wyoming. 


\section{Selected Main-Stem Streams}

A technique commonly used for estimating low-flow characteristics at ungaged sites on main-stem streams, especially regulated streams, is interpolation of a graph of the selected characteristic versus distance along the channel. Such a graph was prepared for the 70 , in the main stem of the Powder River and one of its principal tributaries, Clear Creek (fig. 7). Flows in the main stems of streams on the plains normally are depleted by evapotranspiration; in the case of the Powder River, withdrawals for irrigation also are a major reason for the downstream decrease in low flows.

The $70_{1}$ also was plotted against channel distance for the North Platte River (fig. 8). The reach of this stream within the study area is totally regulated. A considerable amount of water is stored temporarily in the alluvium during the high flows of the snowmelt and irrigation seasons, contributing to subsequent low flows. The low flows downstream from Glendo and Guernsey dams are from seepage after the dams are closed. The graphs in figures 7 and 8 can be interpolated by using the channel distance to the site of interest, as measured on a large-scale map.

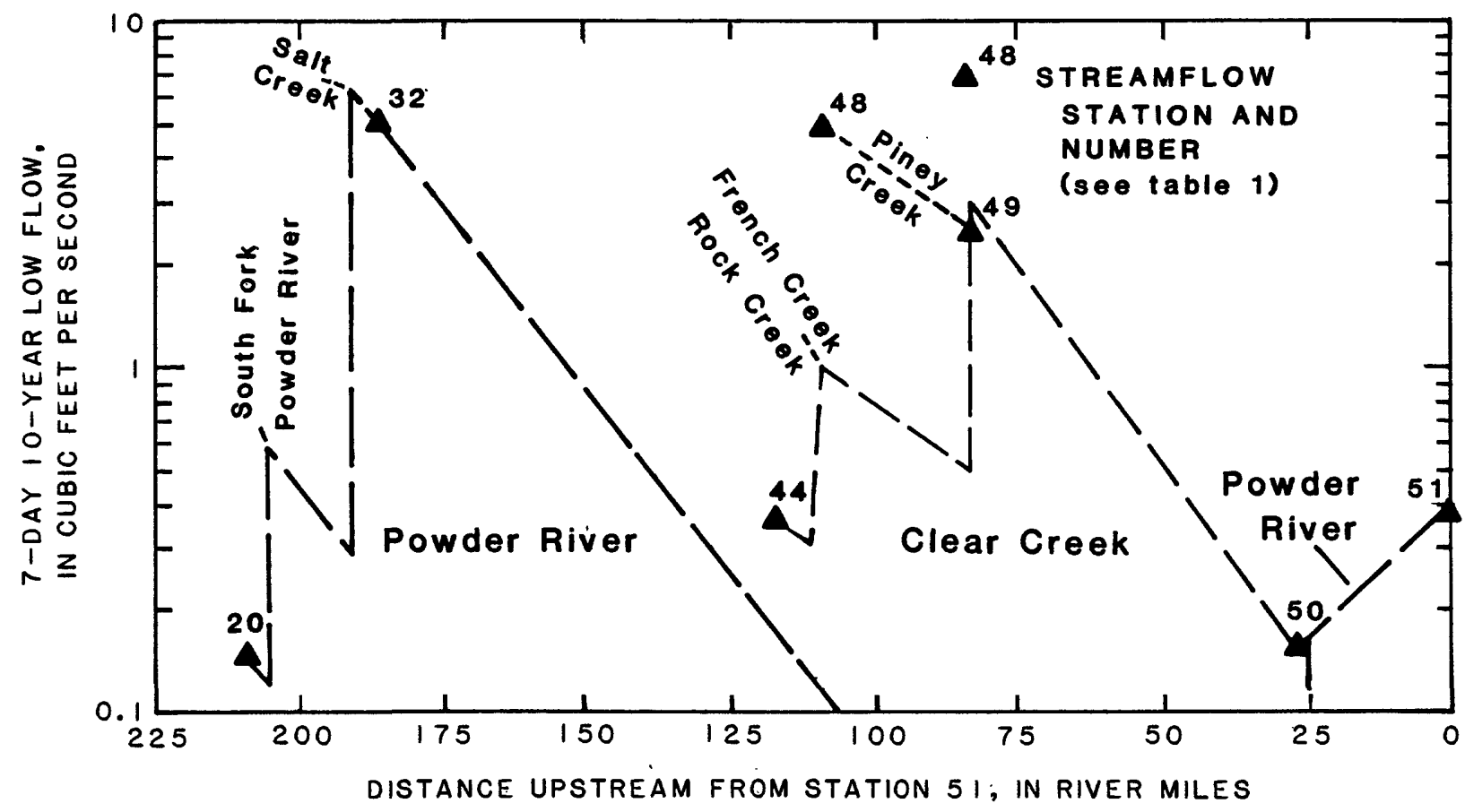

Figure 7.--Relation of 7-day 10-year low flow to distance along channels of the Powder River upstream from Moorhead, Montana, and Clear Creek upstream from its mouth at the Powder River. (Station 41 at river mile 50 not shown. Flow is less than 0.1 cubic foot per second.) 


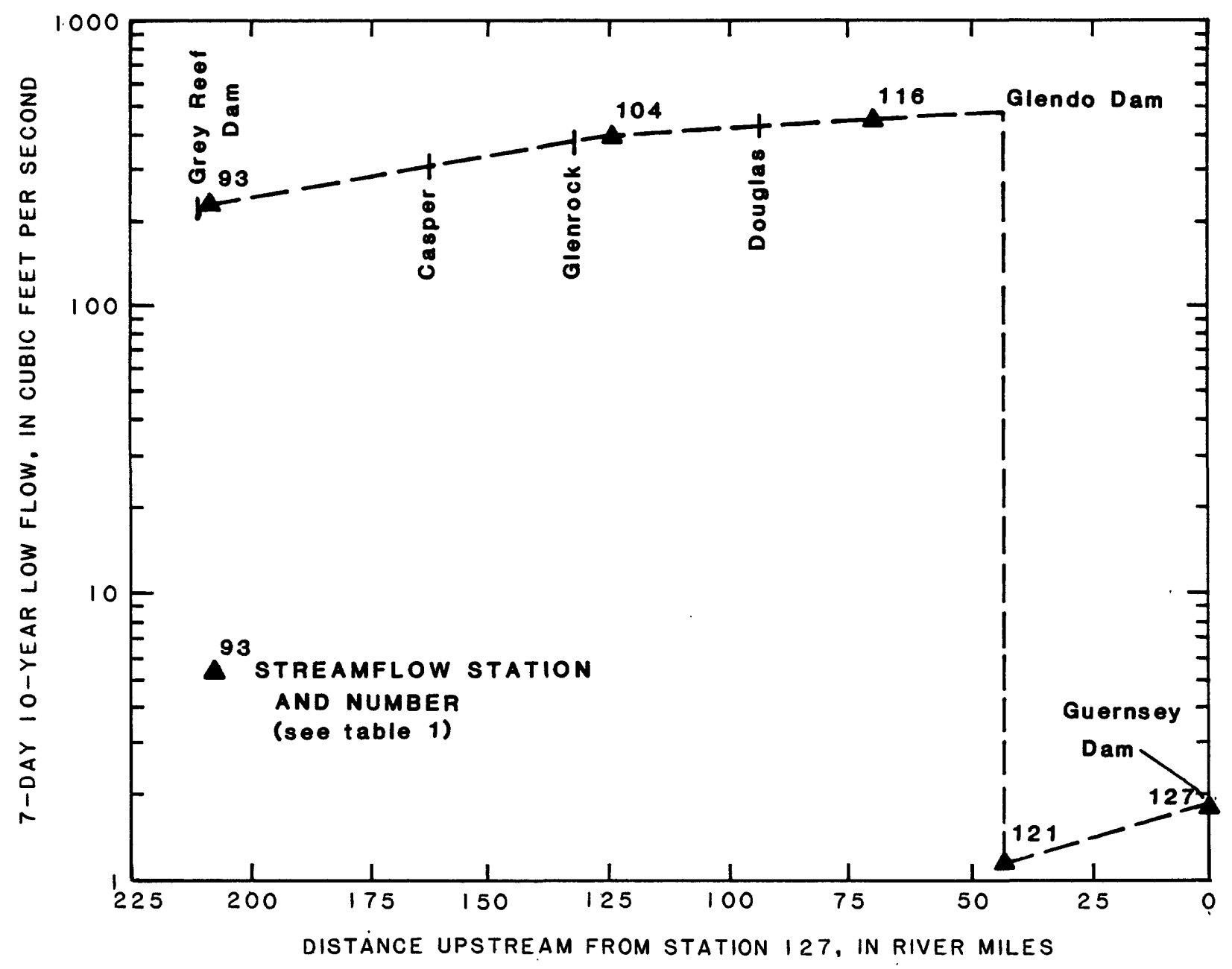

Figure 8.--Relation of 7-day 10-year low flow to distance along channel, North Platte River between Grey Reef Dam and Guernsey Dam, Wyoming. 


\section{DISCUSSION}

\section{Accuracy of Results}

The accuracy of the results described in this report generally cannot be assessed quantitatively. However, the user of the information should be aware of the general accuracy and limitations of the data and techniques used. The following discussion is separated into three sequential categories: (1) Data used, (2) frequency distributions used, and (3) estimating techniques for ungaged sites.

Data errors include streamflow-measurement errors and time-sampling errors. Streamflow measurements and computation of streamflow records were made following well-established procedures of the Geological Survey. Errors in measurements and records computation tend to balance out and are considered to be negligible in comparison with errors in statistical analyses of the data. Of course, the on-site observation of no flow by a hydrographer is without error. Time-sampling errors, on the other hand, may be substantial. As shown by the flow-duration curves (fig. 3), low-flow characteristics not only depend on the length of record, they also are influenced greatly by the inclusion or exclusion of a major drought. Only those stations in operation for 10 or more years between 1930 and about 1960 are considered to have recorded a significant drought. Low-flow characteristics for stations installed since about 1960 (there have been many in northeastern Wyoming) are likely to be much larger than characteristics determined for a period of equal length prior to about 1960. Furthermore, records prior to 1930 were not used because they may be from a different population of streamflow than those since 1930, due to unexplained causes.

The accuracy of frequency distributions is dependent on the timesampling errors described above and on the curve-fitting method used. Regarding assessment of time-sampling errors, in its guidelines on discussing the results of low-flow investigations, the Water Resources Division, U.S. Geological Survey (written commun., 1979) states: "The general relation for estimating the reliability of a point on a frequency curve, in terms of length of record and variability, assumes that the data are completely random. This assumption is not fully acceptable for low flows; * * * Thus, the purely statistical estimate of the reliability of a frequency curve is weak." Such an estimate of reliability was not attempted in this study. Also, the extremes of a frequency curve (probabilities of 5 percent or less, or 90 percent or greater, table 2) may be less reliable than the rest of the curve because of extrapolation, or because the smallest flows may be affected by human activities. For some stations, the log Pearson Type III distribution produces higher or lower estimates of low flows than a simple graphical fit of the data; however, the fit of that distribution to the data between probabilities 5 and 90 percent was very good for most of the stations Iisted in table 2 .

The use of a regression equation to estimate the $7 Q_{10}$ at ungaged sites in northeastern Wyoming is limited to perennial streams in the Bighorn Mountains. Although the equation (fig. 6) has a reasonably small standard error, 64 percent; it should be used with caution. Also, it should be used only for sites having drainage areas of 2 to 200 square miles, which is the 
range of the data used. Likewise, the necessarily straight-line interpolation of the graphs for main stems (figs. 7 and 8 ) should be done with caution. As discussed in the next section, a multiple-regression equation using additional independent variables would improve the accuracy of the estimated characteristics.

\section{Future Investigations}

The results of this investigation indicate a need for future investigations. There are two possible approaches to follow in future investigations: (1) Improvement of the low-flow data base and the information needed to explain quantitatively the causes of variations in low flows; and (2) assessment of the availability of what appear to be marginal quantities of water for human use. Given the predominance of ephemeral streams on the plains of northeastern Wyoming, the improvement of the low-flow information base is attractive from a scientific viewpoint; however, assessing the availabilty of water for storage of meager natural flows may be more important from an economics viewpoint. The two approaches are not mutually exclusive; both require records from streamflow stations and an understanding of the hydrology of small streams in semiarid regions. Hence, both approaches should be considered.

A comprehensive data-collection plan is needed to improve the data base. Such a plan could be manpower-intensive, a definite disincentive. More stations, especially on small plains streams, are needed. Long-term records are essential for sampling low flows over time, but are not necessarily essential for obtaining a large spatial increase in information. The use of lowflow partial-records stations (only high-flow stations have been used in Wyoming to date) would be an inexpensive way to increase the data base substantially. The most important part of the data-collection plan would be obtaining baseflow measurements at as many sites on as many streams as possible, for correlation with long-term baseflow at continuous-record stations. Some baseflow measurements should be planned as seepage runs that will provide information about gains from or losses to ground water. Waterquality measurements (temperature and specific conductance) should be obtained along with the discharge measurements, for possible indications of the geology or of the effect of human activity. The details of comprehensive low-flow data collection are described by Riggs (1972, p. 15-16).

Synoptic baseflow information, as described above, probably would provide a sound basis for estimating low-flow characteristics at ungaged sites, with or without using regression. However, the regression approach, in spite of its limitations for estimating low flows, would be enhanced by the expanded data base and by using multiple regression instead of the twovariable regression used in this report. Standard errors would be improved substantially by using several carefully researched independent variables-basin and meteorological characteristics and, if possible, a characteristic based on geology. 
A potentially more accurate method (Stedinger and Thomas, 1985) uses baseflow measurements at an ungaged site to establish a regression relationship with low flows at a nearby streamflow station. The mean and standard deviation of annual low flows at the ungaged site are estimated from the regression and used to determine low-flow frequency information at the ungaged site. This method, as well as the one described in the preceding paragraph, apply only to perennial and intermittent streams.

Potential long-term supplies of water can be estimated by applying a mathematical technique called storage analysis. Storage analysis provides the probability that a hypothetical reservoir will be unable to provide a specified downstream water supply. The procedures for storage analysis of perennial streams (mainly the mountain streams in northeastern Wyoming) are described by Riggs and Hardison (1973). This might be done for streams that otherwise are fully appropriated. Glover (1984) developed a model for applying storage analysis to ephemeral streams that are typical of the plains areas. Streamflow-station records are required for both procedures. The use of these procedures should be considered for future investigations. 
Bille, Ed, 1978, Early days at Salt Creek and Teapot Dome: Casper, Wyo., Mountain States Lithograph Co., p. 80.

Bingham, R.H., 1982, Low-flow characteristics of Alabama streams: U.S. Geological Survey Water-Supply Paper 2083, 27 p.

Chang, Mingteh, and Boyer, D.G., 1977, Estimates of low flows using watershed and climatic parameters: Water Resources Research, v. 13, no. 6, p. 9971001 .

Chiang, Sie Ling, and Johnson, F.W., 1976, Low flow criteria for diversions and impoundments: American Society of Civil Engineers, Journal of the Water Resources Planning and Management Division, v. 102, no. WR2, p. 227238.

Druse, S.A., Dodge, K.A., and Hotchkiss, W.R., 1981, Base flow and chemical quality of streams in the northern Great Plains area, Montana and Wyoming, 1977-78: U.S. Geological Survey Water-Resources Investigations Open-File Report 81-692, 60 p.

Furness, L.W., 1960, Kansas streamflow characteristics, part 2, low-flow frequency: Kansas Water Resources Board Technical Report 2, 179 p.

Furness, L.W., and Busby, M.W., 1967, Two methods of estimating base flow at ungaged stream sites in Kansas and adjacent states, in Geological Survey Research 1967: U.S. Geological Survey Professional Paper 575-C, p. C208C211.

Glover, K.C., 1984, Storage analysis for ephemeral streams in semiarid regions: U.S. Geological Survey Water-Resources Investigations Report 834078, 55 p.

Hodson, W.G., Pearl, R.H., and Druse, S.A., 1973, Water resources of the Powder River basin and adjacent areas, northeastern Wyoming: U.S. Geological Survey Hydrologic Investigations Atlas HA-465, 4 sheets.

Huff, F.A., and Changenon, S.A., Jr., 1964, Relation between precipitation deficiency and low streamflow: Journal of Geophysical Research, v. 69, no. 1 , p. 605-613.

Huntzinger, T.L., 1978, Low-flow characteristics of Oklahoma streams: U.S. Geological Survey Open-File Report 78-166, 93 p.

Institute of Hydrology, 1979, Catchment characteristic estimation manual, in Low-flow studies report: Crowmarsh Gifford, Wallingford, Oxon, United Kingdam, Institute of Hydrology Report 3, 26 p.

Lara, O.G., 1979, Annual and seasonal low-flow characteristics of Iowa streams: U.S. Geological Survey Open-File Report 79-555, 55 p. 
Lenfest, L.W., Jr., 1986, Evapotranspiration rates at selected sites in the Powder River basin, Wyoming and Montana: U.S. Geological Survey WaterResources Investigations Report 82-4105, 23 p.

Lowham, H.W., 1976, Techniques for estimating flow characteristics of Wyaming streams: U.S. Geological Survey Water-Resources Investigations Report 76$112,83 \mathrm{p}$.

Lowry, M.E., Wilson, J.F., Jr., and others, 1986, Hydrology of Area 50, Northern Great Plains and Rocky Mountain Coal Regions, Wyoming and Montana: U.S. Geological Survey Water-Resources Investigations Open-File Report 83-545, 137 p.

Matalas, N.C., 1963, Probability distribution of low flows: U.S. Geological Survey Professional Paper 434-A, 27 p.

Matthai, H.F., 1979, Hydrologic and human aspects of the 1976-77 drought: U.S. Geological Survey Professional Paper 1130, 84 p.

Riggs, H.C., 1972, Low-flow investigations: U.S. Geological Survey Techniques of Water-Resources Investigations, book 4, chap. Bl, 18 p.

1980, Characteristics of low flows: American Society of civil Engineers, Journal of the Hydraulics Division, v. 106, no. HY5, p. 717-731.

Riggs, H.C., and Hardison, C.H., 1973, Storage analysis for water supply: U.S. Geological Survey Techniques of Water-Resources Investigations, book 4, chap. B2, 20 p.

Searcy, J.K., 1959, Flow-duration curves: U.S. Geological Survey Water-Supply Paper 1542-A, 33 p.

Sen, Zekai, 1980, Statistical analysis of hydrologic critical droughts: American Society of Civil Engineers, Journal of the Hydraulics Division, v. 106, no. HYl, p. 99-115.

Singh, K.P., 1969, Theoretical baseflow curves: American Society of Civil Engineers, Journal of the Hydraulics Division, v. 95, no. HY6, p. 20292048.

Singh, K.P., and Stall, J.B., 1974, Hydrology of 7-day 10-year low-flows: American Society of Civil Engineers, Journal of the Hydraulics Division, v. 100, no. HYl2, p. 1753-1771.

Skelton, John, 1974, Estimating low-flow frequency for perennial Missouri Ozarks streams: U.S. Geological Survey Water-Resources Investigations Report 59-73, 25 p.

Speer, P.R., Golden, H.G., Patterson, J.F., and others, 1964, Low-flow characteristics of streams in the Mississippi Embayment in Mississippi and Alabama, with a section on Quality of the water, by W.J. Welborne: U.S. Geological Survey Professional Paper 448-I, 47 p. 
Speer, P.R., Perry, W.J., McCabe, J.A., Lara, O.G., and others, 1965, Low-flow characteristics of streams in the Mississippi Embayment in Tennessee, Kentucky, and Illinois, with a section on Quality of the water, by H.G. Jeffery: U.S. Geological Survey Professional Paper 448-H, 36 p.

Stedinger, J.R., and Thomas, W.O., Jr., 1985, Low-flow frequency estimation using base-flow measurements: U.S. Geological Survey Open-File Report $85-95,22$ p.

Wahl, K.L., 1970, A proposed streamflow data program for Wyoming: U.S. Geological Survey open-file report, 44 p.

Whipple, William, Jr., 1966, Regional drought frequency analysis: American Society of Civil Engineers, Journal of the Irrigation and Drainage Division, v. 92, no. IR2, p. 11-31. 